\title{
Certain Energies of Graphs for Dutch Windmill and Double-Wheel Graphs
}

\author{
Jing Wu $\mathbb{D},{ }^{1}$ Muhammad Arfan Ali, ${ }^{2}$ Hafiz Mutee ur Rehman $\mathbb{D},{ }^{3}$ and Yan Dou ${ }^{4}$ \\ ${ }^{1}$ School of Science, Xijing University, Xi'an 710123, China \\ ${ }^{2}$ Virtual University of Pakistan, 54-Lawrence Road, Lahore, Pakistan \\ ${ }^{3}$ Department of Mathematics, Division of Science and Technology, University of Education, Lahore 54000, Pakistan \\ ${ }^{4}$ Xinjiang Normal University, The Elementary Education College, \\ The Children's Education Practice Innovation Research Center in Xinjiang, Urumqi 830000, China
}

Correspondence should be addressed to Jing Wu; specialwujing@sina.com and Hafiz Mutee ur Rehman; mutee.rehman@ue.edu.pk

Received 14 October 2021; Revised 23 November 2021; Accepted 29 November 2021; Published 14 February 2022

Academic Editor: Gul Rahmat

Copyright (c) 2022 Jing Wu et al. This is an open access article distributed under the Creative Commons Attribution License, which permits unrestricted use, distribution, and reproduction in any medium, provided the original work is properly cited.

Energy of a graph is defined as the sum of the absolute values of the eigenvalues of the adjacency matrix associated with the graph. In this research work, we find color energy, distance energy, Laplacian energy, and Seidel energy for the Dutch windmill graph of cycle lengths 4, 5, and 6. Also, we find the lower bounds of the double-wheel graph for energy, Seidel energy, color energy, distance energy, Laplacian energy, and Harary energy.

\section{Introduction}

Energy of a graph is one of the important concepts in graph theory. The energy of a graph is mostly studied in the context of spectral graph theory. Gutman [1] introduced the concept of energy of a graph for the first time. Gutman defined energy of a graph as the sum of the absolute values of the eigenvalues of the adjacency matrix associated with the graph. Adjacency matrix is used to represent a finite graph, and it is a square matrix. The entries of the adjacency matrix provide information whether any two vertices are joined with each other or not. The total pi electron energy of conjugated hydrocarbons in chemistry computed by using Huckel molecular orbital theory coincides with the energy defined by Gutman, so the energies calculated in graph theory have a special significance.

Calculating the bounds for graph spectra has been the main area of research among graph theorists. McClelland [2] worked on the estimation of pi electron energies. Nageswari and Sarasija [3] calculated edge energy bounds of finite, simple, and undirected graphs. Das and Gutman [4] calculated some upper and lower bounds for $E(G)$ in terms of number of vertices and number of edges. Adiga and Rakshith [5] calculated upper bounds for the extended energy of graphs. Jahanbani [6] calculated lower bounds for the energy of graphs. In this research work, we calculate lower bounds for various energies of the double-wheel graph for the first time. Now, we introduce some terminologies associated with our work.

In mathematics, in graph theory, we study mathematical structures which are called graphs, and we use these graphs to represent pairwise relations between different objects. So, graph theory is the study of graphs and their characteristics. One of the most important concepts in graph theory is energy of a graph. This concept was introduced by Gutman [1] which defined energy as the sum of the absolute values of the roots of the characteristics equation, and these roots are called eigenvalues of the graph under consideration.

In [7], energy, Seidel energy, Harary energy, distance energy, color energy, and Laplacian energy of the friendship graph have been calculated. In this research paper, we calculate these energies of the Dutch windmill graph of cycle lengths 4, 5, and 6, and also, we find lower bounds of these energies of the double-wheel graph. 


\section{Basic Definitions and Notations}

Definition 1. For a graph $G$, the adjacency matrix is the square matrix of order $n \times n$ denoted by $A(G)$ and is defined as $A(G)=\left[a_{i j}\right]$, where

$$
a_{i j}= \begin{cases}1 & \text { if } v_{i} v_{j} \in E \\ 0 & \text { if } v_{i} v_{j} \notin E .\end{cases}
$$

The adjacency matrix gives us information whether a vertex in a pair of vertices is joined with the other vertex or not.
Definition 2. The Laplacian matrix is also a square matrix defined as follows:

$$
L a(G)=D E G(G)-A(G),
$$

where $D E G(G)$ is the diagonal matrix of vertex degrees and $A(G)$ is the adjacency matrix of graph $G$.

Definition 3. The Harary matrix of a graph $G$ is the square matrix of order $n$ whose $(i, j)$ entry is defined as $\left(1 / d_{i j}\right)$ where $d_{i j}$ is the distance between the vertices $v_{i}$ and $v_{j}$.

Definition 4. The color matrix of $G$ is the square matrix of order $n$ denoted by $A_{c}(G)=\left[a_{i j}\right]$ where

$$
a_{i j}= \begin{cases}1 & \text { if } v_{i} \text { and } v_{j} \text { are adjacent with different colors } \\ -1 & \text { if } v_{i} \text { and } v_{j} \text { are nonadjacent with the same color } \\ 0, & \text { otherwise. }\end{cases}
$$

Definition 5. The distance matrix of $G$ is a matrix of order $n$, and its entries represent shortest distances between its different vertices.

Definition 6. The Seidel matrix of a graph is denoted by $S(G)=a_{i j}$, where

$$
a_{i j}= \begin{cases}1 & \text { if } v_{i} v_{j} \notin E \\ -1 & \text { if } v_{i} v_{j} \in E \\ 0, & \text { otherwise. }\end{cases}
$$

The Seidel matrix is a square, real symmetric matrix of order $n$. The eigenvalues of the Seidel matrix are the eigenvalues of the graph $G$.

Definition 7. The sum of the absolute values of the roots of the characteristic equation associated with the adjacency matrix of the graph determines the energy of that graph. If $\lambda_{1}, \lambda_{2}, \lambda_{3}, \ldots, \lambda_{n}$ are the eigenvalues of the adjacency matrix, then energy of graph $E(G)$ is defined as follows:

$$
E(G)=\sum_{i=1}^{n}\left|\lambda_{i}\right|
$$

Haemers [7] introduced Seidel energy of a graph. To calculate the Seidel energy of a graph, we make its Seidel matrix as defined in equation (4) and find its characteristic equation; now, the sum of the absolute values of the roots of this characteristic equation determines the Seidel energy of the graph under consideration.

Definition 8. If $\lambda_{1}, \lambda_{2}, \lambda_{3}, \ldots, \lambda_{n}$ are the eigenvalues of the Seidel matrix, then Seidel energy is defined as follows:

$$
E(G)=\left|\lambda_{1}\right|+\left|\lambda_{2}\right|+\left|\lambda_{3}\right|+\cdots+\left|\lambda_{n}\right|
$$

Zhou [8] introduced Laplacian energy of a graph. To calculate Laplacian energy of a graph, first, we form its Laplacian matrix as defined in 2.3.7; then, we find its characteristic equation. Now, the sum of the absolute values of the roots of this characteristic equation determines the Laplacian energy of the graph under consideration.

Definition 9. If $\lambda_{1}, \lambda_{2}, \lambda_{3}, \ldots, \lambda_{n}$ are the eigenvalues of the Laplacian matrix, then Laplacian energy is defined as follows:

$$
\operatorname{L.E}(G)=\left|\lambda_{1}\right|+\left|\lambda_{2}\right|+\left|\lambda_{3}\right|+\cdots+\left|\lambda_{n}\right| .
$$

Indulal et al. [9] defined distance energy of a graph. To calculate distance energy of a graph, first, we form its distance matrix as defined in 2.3.3; then, we find its characteristic equation. Now, the sum of the absolute values of the roots of this characteristic equation determines the distance energy of the graph under consideration.

Definition 10. If $\lambda_{1}, \lambda_{2}, \lambda_{3}, \ldots, \lambda_{n}$ are the eigenvalues of the distance matrix, then distance energy D.E $(G)$ is defined as follows:

$$
\operatorname{D.E}(G)=\left|\lambda_{1}\right|+\left|\lambda_{2}\right|+\left|\lambda_{3}\right|+\cdots+\left|\lambda_{n}\right| \text {. }
$$

Color energy was defined by Adiga and Smitha [10]. To calculate color energy of a graph, first, we form its color matrix, and then we find its characteristic equation. Now, the sum of the absolute values of the roots of this characteristic equation determines the color energy of the graph under consideration.

Definition 11. If $\lambda_{1}, \lambda_{2}, \lambda_{3}, \ldots, \lambda_{n}$ are the eigenvalues of the color matrix, then color energy is defined as follows:

$$
\text { C.E }(G)=\left|\lambda_{1}\right|+\left|\lambda_{2}\right|+\left|\lambda_{3}\right|+\cdots+\left|\lambda_{n}\right| .
$$

Harary energy of a graph was defined in [11-13]. To calculate Harary energy of a graph, first, we form its Harary matrix, and then we find its characteristic equation. Now, the sum of the absolute values of the roots of this characteristic equation determines the Harary energy of the graph under 
consideration. For other useful results, we refer to the readers [14-17].

Definition 12. If $\lambda_{1}, \lambda_{2}, \lambda_{3}, \ldots, \lambda_{n}$ are the eigenvalues of the Harary matrix, then Harary energy is defined as follows:

$$
\operatorname{H.E}(G)=\left|\lambda_{1}\right|+\left|\lambda_{2}\right|+\left|\lambda_{3}\right|+\cdots+\left|\lambda_{n}\right| .
$$

\section{Main Results}

Theorem 1. The energy of a Dutch windmill graph $D_{4}^{m}$ is $2(\sqrt{2} m-\sqrt{2}+\sqrt{2 m+2})$.

Proof. The adjacency matrix for $D_{4}^{m}$ is

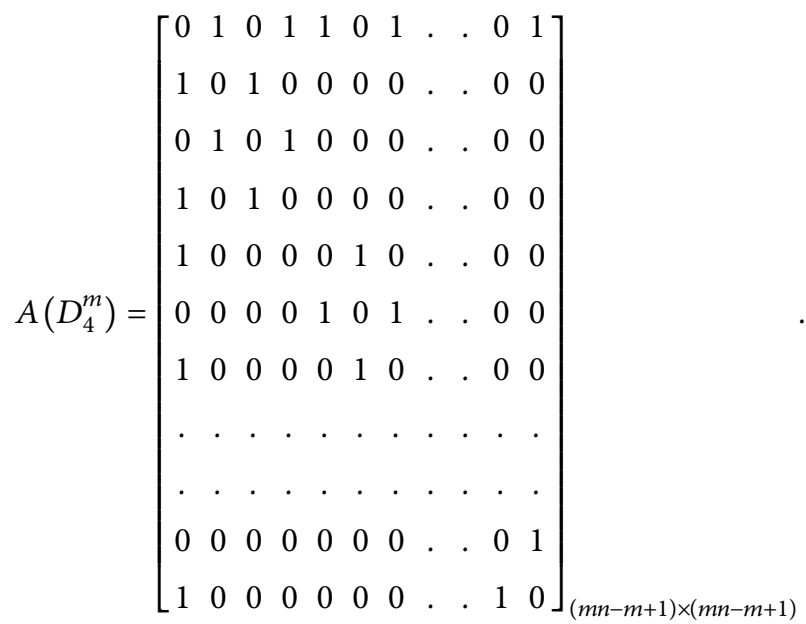

The characteristic equation is $(\lambda-0)^{m+1}\left(\lambda^{2}-2\right)^{m-1}$ $\left(\lambda^{2}-(2 m+2)\right)=0$.

The eigenvalues are $\lambda_{1}=0(m+1$ times $), \lambda_{2}=\sqrt{2}(m$ -1times), $\quad \lambda_{3}=-\sqrt{2}(m-1$ times $), \quad \lambda_{4}=\sqrt{2 m+2}, \quad$ and $\lambda_{5}=-\sqrt{2 m+2}$.

Now, energy of $D_{4}^{m}$ can be calculated by using Definition 2.4.1 as follows:

$$
\begin{aligned}
E\left(D_{4}^{m}\right)= & (m+1)\left|\lambda_{1}\right|+(m-1)\left|\lambda_{2}\right|+(m-1)\left|\lambda_{3}\right|+\left|\lambda_{4}\right|+\left|\lambda_{5}\right| \\
= & (m+1)|0|+(m-1)|\sqrt{2}|+(m-1) \mid \\
& -\sqrt{2}|+| \sqrt{2 m+2}|+|-\sqrt{2 m+2} \mid
\end{aligned}
$$

$$
\begin{aligned}
& =\sqrt{2} m-\sqrt{2}+\sqrt{2} m-\sqrt{2}+\sqrt{2 m+2}+\sqrt{2 m+2} \\
& =2(\sqrt{2} m-\sqrt{2}+\sqrt{2 m+2}) .
\end{aligned}
$$

Theorem 2. The energy of $D_{5}^{m}$ is $2 \sqrt{5} m-\sqrt{5}+\chi$.

Proof. The adjacency matrix for $D_{5}^{m}$ is

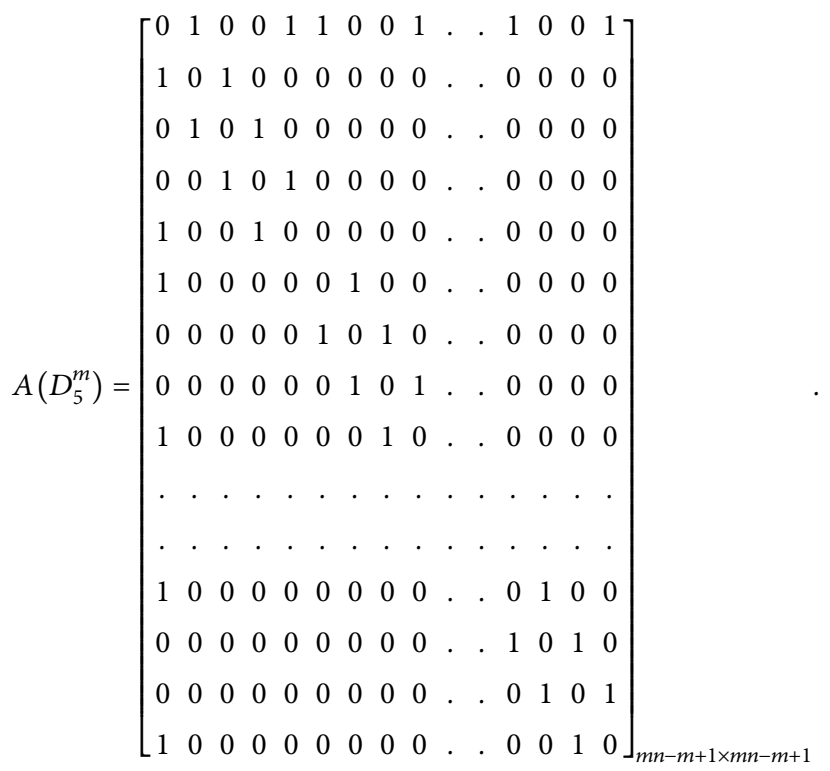

The characteristic equation of the above adjacency matrix is

$$
\begin{aligned}
& \left(\lambda^{2}-\lambda-1\right)^{m-1}\left(\lambda^{2}+\lambda-1\right)^{m} \\
& \quad \cdot\left(\lambda^{3}-\lambda^{2}-(2 m+1) \lambda+2 m\right)=0
\end{aligned}
$$

The eigenvalues are $\lambda_{1}=(-1+\sqrt{5} / 2)$ ( $m$ times), $\lambda_{2}=$ $(-1-\sqrt{5} / 2)(m$ times $), \lambda_{3}=(1-\sqrt{5} / 2)(m-1$ times $), \lambda_{4}=$ $(1+\sqrt{5} / 2)(m-1$ times $)$, and $\lambda_{5}, \lambda_{6}, \lambda_{7}$, where $\lambda_{5}, \lambda_{6}, \lambda_{7}$ are the roots of the cubic equation given in the following:

$$
\lambda^{3}-\lambda^{2}-(2 m+1) \lambda+2 m=0
$$

Now, energy can be calculated by using Definition 2.4.1 as follows:

$$
\begin{aligned}
& E\left(D_{5}^{m}\right)=m\left|\lambda_{1}\right|+m\left|\lambda_{2}\right|+(m-1)\left|\lambda_{3}\right|+(m-1)\left|\lambda_{4}\right|+\left|\lambda_{5}\right|+\left|\lambda_{6}\right|+\left|\lambda_{7}\right|, \\
& E\left(D_{5}^{m}\right)=m\left|\frac{-1+\sqrt{5}}{2}\right|+m\left|\frac{-1-\sqrt{5}}{2}\right|+(m-1)\left|\frac{1-\sqrt{5}}{2}\right|+(m-1)\left|\frac{1+\sqrt{5}}{2}\right|+\left|\lambda_{5}\right|+\left|\lambda_{6}\right|+\left|\lambda_{7}\right|, \\
& E\left(D_{5}^{m}\right)=m\left|\frac{-1+\sqrt{5}}{2}\right|+m\left|\frac{-1-\sqrt{5}}{2}\right|+(m-1)\left|\frac{1-\sqrt{5}}{2}\right|+(m-1)\left|\frac{1+\sqrt{5}}{2}\right|+\chi, \\
& E\left(D_{5}^{m}\right)=2 \sqrt{5} m-\sqrt{5}+\chi \\
& \quad \chi=\left|\lambda_{5}\right|+\left|\lambda_{6}\right|+\left|\lambda_{7}\right| .
\end{aligned}
$$


Theorem 3. The energy of a Dutch windmill graph $D_{6}^{m}$ is

$$
2(1+\sqrt{3}) m-2 \sqrt{3}+2 \sqrt{\frac{(2 m+3)+\sqrt{4 m^{2}-4 m+9}}{2}}+2 \sqrt{\frac{(2 m+3)-\sqrt{4 m^{2}-4 m+9}}{2}} .
$$

Proof. The adjacency matrix for $D_{6}^{m}$ is

$$
A\left(D_{6}^{m}\right)=\left[\begin{array}{llllllllllllllllll}
0 & 1 & 0 & 0 & 0 & 1 & 1 & 0 & 0 & 0 & 1 & . & . & 1 & 0 & 0 & 0 & 1 \\
1 & 0 & 1 & 0 & 0 & 0 & 0 & 0 & 0 & 0 & 0 & . & . & 0 & 0 & 0 & 0 & 0 \\
0 & 1 & 0 & 1 & 0 & 0 & 0 & 0 & 0 & 0 & 0 & . & . & 0 & 0 & 0 & 0 & 0 \\
0 & 0 & 1 & 0 & 1 & 0 & 0 & 0 & 0 & 0 & 0 & . & . & 0 & 0 & 0 & 0 & 0 \\
0 & 0 & 0 & 1 & 0 & 1 & 0 & 0 & 0 & 0 & 0 & . & . & 0 & 0 & 0 & 0 & 0 \\
1 & 0 & 0 & 0 & 1 & 0 & 0 & 0 & 0 & 0 & 0 & . & . & 0 & 0 & 0 & 0 & 0 \\
1 & 0 & 0 & 0 & 0 & 0 & 0 & 1 & 0 & 0 & 0 & . & . & 0 & 0 & 0 & 0 & 0 \\
0 & 0 & 0 & 0 & 0 & 0 & 1 & 0 & 1 & 0 & 0 & . & . & 0 & 0 & 0 & 0 & 0 \\
0 & 0 & 0 & 0 & 0 & 0 & 0 & 1 & 0 & 1 & 0 & . & . & 0 & 0 & 0 & 0 & 0 \\
0 & 0 & 0 & 0 & 0 & 0 & 0 & 0 & 1 & 0 & 1 & . & . & 0 & 0 & 0 & 0 & 0 \\
1 & 0 & 0 & 0 & 0 & 0 & 0 & 0 & 0 & 1 & 0 & . & . & 0 & 0 & 0 & 0 & 0 \\
. & . & . & . & . & . & . & . & . & . & . & . & . & . & . & . & . & . \\
0 & . & . & . & . & . & . & . & . & . & . & . & . & . & . & . & . & . \\
1 & 0 & 0 & 0 & 0 & 0 & 0 & 0 & 0 & 0 & 0 & . & . & 0 & 1 & 0 & 0 & 0 \\
0 & 0 & 0 & 0 & 0 & 0 & 0 & 0 & 0 & 0 & 0 & . & . & 1 & 0 & 1 & 0 & 0 \\
0 & 0 & 0 & 0 & 0 & 0 & 0 & 0 & 0 & 0 & 0 & . & . & 0 & 1 & 0 & 1 & 0 \\
0 & 0 & 0 & 0 & 0 & 0 & 0 & 0 & 0 & 0 & 0 & . & . & 0 & 0 & 1 & 0 & 1 \\
1 & 0 & 0 & 0 & 0 & 0 & 0 & 0 & 0 & 0 & 0 & . & . & 0 & 0 & 0 & 1 & 0
\end{array}\right]_{m n-m+1 \times m n-m+1}
$$

The characteristic equation is $(\lambda+1)^{m}(\lambda-1)^{m}(\lambda-$ $0)^{m-1}\left(\lambda^{2}-3\right)^{m-1}\left(\lambda^{4}-(2 m+3) \lambda^{2}+4 m\right)=0$.

The eigenvalues of the above characteristic equation are $\lambda_{1}=-1$ ( $m$ times $), \quad \lambda_{2}=1$ ( $m$ times $), \quad \lambda_{3}=0$ ( $m-1$ times $)$ $\lambda_{4}=\sqrt{3}(m-1$ times $), \quad \lambda_{5}=-\sqrt{3}(m-1$ times $), \quad \lambda_{6}=$ $\sqrt{\left((2 m+3)+\sqrt{4 m^{2}-4 m+9} / 2\right)}, \quad \lambda_{7}=$ $\sqrt{\left((2 m+3)-\sqrt{4 m^{2}-4 m+9} / 2\right)}, \quad \lambda_{8}=-$ $\sqrt{\left((2 m+3)+\sqrt{4 m^{2}-4 m+9} / 2\right)}, \quad$ and $\quad \lambda_{9}=-$ $\sqrt{\left((2 m+3)-\sqrt{4 m^{2}-4 m+9} / 2\right)}$.

Now, energy can be calculated by using Definition 2.4.1 as follows: 


$$
\begin{aligned}
E\left(D_{6}^{m}\right)= & m\left|\lambda_{1}\right|+m\left|\lambda_{2}\right|+(m-1)\left|\lambda_{3}\right|+(m-1)\left|\lambda_{4}\right|+(m-1)\left|\lambda_{5}\right|+\left|\lambda_{6}\right|+\left|\lambda_{7}\right|+\left|\lambda_{8}\right|+\left|\lambda_{9}\right|, \\
= & m|-1|+m|1|+(m-1)|0|+(m-1)|\sqrt{3}|+(m-1)|-\sqrt{3}|+\left|\sqrt{\frac{(2 m+3)+\sqrt{4 m^{2}-4 m+9}}{2}}\right|+\left|\sqrt{\frac{(2 m+3)-\sqrt{4 m^{2}-4 m+9}}{2}}\right|+\left|-\sqrt{\frac{(2 m+3)-\sqrt{4 m^{2}-4 m+9}}{2}}\right|, \\
& +\left|-\sqrt{\frac{(2 m+3)+\sqrt{4 m^{2}-4 m+9}}{2}}\right| \\
= & 2(1+\sqrt{3}) m-2 \sqrt{3}+2 \sqrt{\frac{(2 m+3)+\sqrt{4 m^{2}-4 m+9}}{2}}+2 \sqrt{\frac{(2 m+3)-\sqrt{4 m^{2}-4 m+9}}{2} .}
\end{aligned}
$$

Theorem 4. Let $G$ be a double-wheel graph; then, $E(G)>2$ $+2 \sqrt{1+2 n}$.
Proof. The adjacency matrix of order $2 n+1$ for a doublewheel graph $W_{n}, n \geq 3$, is

$$
A\left(W_{n}\right)=\left[\begin{array}{cccccccccccccc}
0 & 1 & 0 & . & . & . & 1 & 0 & 0 & . & . & . & 0 & 1 \\
1 & 0 & 1 & . & . & . & 0 & 0 & 0 & . & . & . & 0 & 1 \\
1 & 1 & 0 & . & . & . & 0 & 0 & 0 & . & . & . & 0 & 1 \\
. & . & . & . & . & . & . & . & . & . & . & . & . & . \\
. & . & . & . & . & . & . & . & . & . & . & . & . & . \\
. & . & . & . & . & . & . & . & . & . & . & . & . & . \\
1 & 0 & 0 & . & . & . & 0 & 0 & 0 & . & . & . & 0 & 1 \\
0 & 0 & 0 & . & . & . & 0 & 0 & 1 & . & . & . & 1 & 1 \\
0 & 0 & 0 & . & . & . & 0 & 1 & 0 & . & . & . & 0 & 1 \\
. & . & . & . & . & . & . & . & . & . & . & . & . & . \\
. & . & . & . & . & . & . & . & . & . & . & . & . & . \\
. & . & . & . & . & . & . & . & . & . & . & . & . & . \\
0 & 0 & 0 & . & . & . & 0 & 1 & 0 & . & . & . & 0 & 1 \\
1 & 1 & 1 & 1 & 1 & 1 & 1 & 1 & 1 & 1 & 1 & 1 & 1 & 0
\end{array}\right]_{2 n+1 \times 2 n+1}
$$

The characteristic equation of the above adjacency matrix is

$$
(\lambda-2)\left(\lambda^{2}-2 \lambda-2 n\right)\left(\lambda^{2 n-2}+\lambda^{2 n-1}+\cdots \lambda\right)=0
$$

The three roots of the above equation are $\lambda_{1}=2, \lambda_{2}=$ $1+\sqrt{1+2 n}$, and $\lambda_{3}=1-\sqrt{1+2 n}$.

Now, the energy of the double-wheel graph can be calculated by using Definition 2.4.1 as follows:

$$
\begin{aligned}
E\left(W_{n}\right)= & \left|\lambda_{1}\right|+\left|\lambda_{2}\right|+\left|\lambda_{3}\right|+\cdots+\left|\lambda_{2 n+1}\right| \\
& >\left|\lambda_{1}\right|+\left|\lambda_{2}\right|+\left|\lambda_{3}\right|
\end{aligned}
$$

$$
\begin{aligned}
& =2+1+\sqrt{1+2 n}-1+\sqrt{1+2 n} \\
& =2+2 \sqrt{1+2 n} \\
& =2(1+\sqrt{1+2 n}) .
\end{aligned}
$$

Theorem 5. The Seidel energy of $D_{4}^{m}$ is $4 \sqrt{2} m-4 \sqrt{2}+m+\chi$.

Proof. The Seidel matrix for $D_{4}^{m}$ is 


$$
S\left(D_{4}^{m}\right)=\left[\begin{array}{ccccccccccccccc}
0 & -1 & 1 & -1 & -1 & 1 & -1 & -1 & 1 & -1 & & -1 & 1 & -1 \\
-1 & 0 & -1 & 1 & 1 & 1 & 1 & 1 & 1 & 1 & & 1 & 1 & 1 \\
1 & -1 & 0 & -1 & 1 & 1 & 1 & 1 & 1 & 1 & & 1 & 1 & 1 \\
-1 & 1 & -1 & 0 & 1 & 1 & 1 & 1 & 1 & 1 & . & . & 1 & 1 & 1 \\
-1 & 1 & 1 & 1 & 0 & -1 & 1 & 1 & 1 & 1 & . & . & 1 & 1 & 1 \\
1 & 1 & 1 & 1 & -1 & 0 & -1 & 1 & 1 & 1 & . & . & 1 & 1 & 1 \\
-1 & 1 & 1 & 1 & 1 & -1 & 0 & 1 & 1 & 1 & . & . & 1 & 1 & 1 \\
1 & 1 & 1 & 1 & 1 & 1 & 1 & 0 & -1 & 1 & . & . & 1 & 1 & 1 \\
-1 & 1 & 1 & 1 & 1 & 1 & 1 & 1 & -1 & 0 & . & . & 1 & 1 & 1 \\
. & . & . & . & . & . & . & . & . & . & . & . & . & . & . \\
. & . & . & . & . & . & . & . & . & . & . & . & . & . & . \\
-1 & 1 & 1 & 1 & 1 & 1 & 1 & 1 & 1 & 1 & . & . & 0 & -1 & 1 \\
1 & 1 & 1 & 1 & 1 & 1 & 1 & 1 & 1 & 1 & . & . & -1 & 0 & -1 \\
-1 & 1 & 1 & 1 & 1 & 1 & 1 & 1 & 1 & . & . & 1 & -1 & 0
\end{array}\right]_{m n-m+1 \times m n-m+1}
$$

The characteristic equation of the above Seidel matrix is

$$
(\lambda+1)^{m}\left(\lambda^{2}+2 \lambda-7\right)^{m-1}\left(\lambda^{3}-(3 m-2) \lambda^{2}+(2 m-7) \lambda+\left(8 m^{2}-11 m\right)\right)=0
$$

Eigenvalues are $\lambda_{1}=-1$ ( $m$ times $), \quad \lambda_{2}=-1+2 \sqrt{2}$ ( $m-1$ times $), \quad \lambda_{3}=-1-2 \sqrt{2}(m-1$ times $)$, and $\lambda_{4}, \lambda_{5}, \lambda_{6}$, where $\lambda_{4}, \lambda_{5}, \lambda_{6}$ are the roots of the cubic equation given in the following:

$$
\left(\lambda^{3}-(3 m-2) \lambda^{2}+(2 m-7) \lambda+\left(8 m^{2}-11 m\right)\right)=0 .
$$

Now, Seidel energy can be calculated by using Definition 2.4.2 as follows:

$$
\begin{aligned}
\text { S.E. }(G) & =m\left|\lambda_{1}\right|+(m-1)\left|\lambda_{2}\right|+(m-1)\left|\lambda_{3}\right|+\left|\lambda_{4}\right|+\left|\lambda_{5}\right|+\left|\lambda_{6}\right| \\
& =m|-1|+(m-1)|-1+2 \sqrt{2}|+(m-1)|-1-2 \sqrt{2}|+\left|\lambda_{4}\right|+\left|\lambda_{5}\right|+\left|\lambda_{6}\right| \\
& =m|-1|+(m-1)|-1+2 \sqrt{2}|+(m-1)|-1-2 \sqrt{2}|+\chi \\
& =m-m+1+2 \sqrt{2} m-2 \sqrt{2}+m-1+2 \sqrt{2} m-2 \sqrt{2}+\chi \\
\chi & =\left|\lambda_{4}\right|+\left|\lambda_{5}\right|+\left|\lambda_{6}\right| \\
& =4 \sqrt{2} m-4 \sqrt{2}+m+\chi, \chi=\left|\lambda_{4}\right|+\left|\lambda_{5}\right|+\left|\lambda_{6}\right| .
\end{aligned}
$$


Theorem 6. The Seidel energy of $D_{5}^{m}$ is given by Proof. The Seidel matrix for $D_{5}^{m}$ is $2[(\sqrt{5}+\sqrt{14}) m-\sqrt{14}]+\chi$.

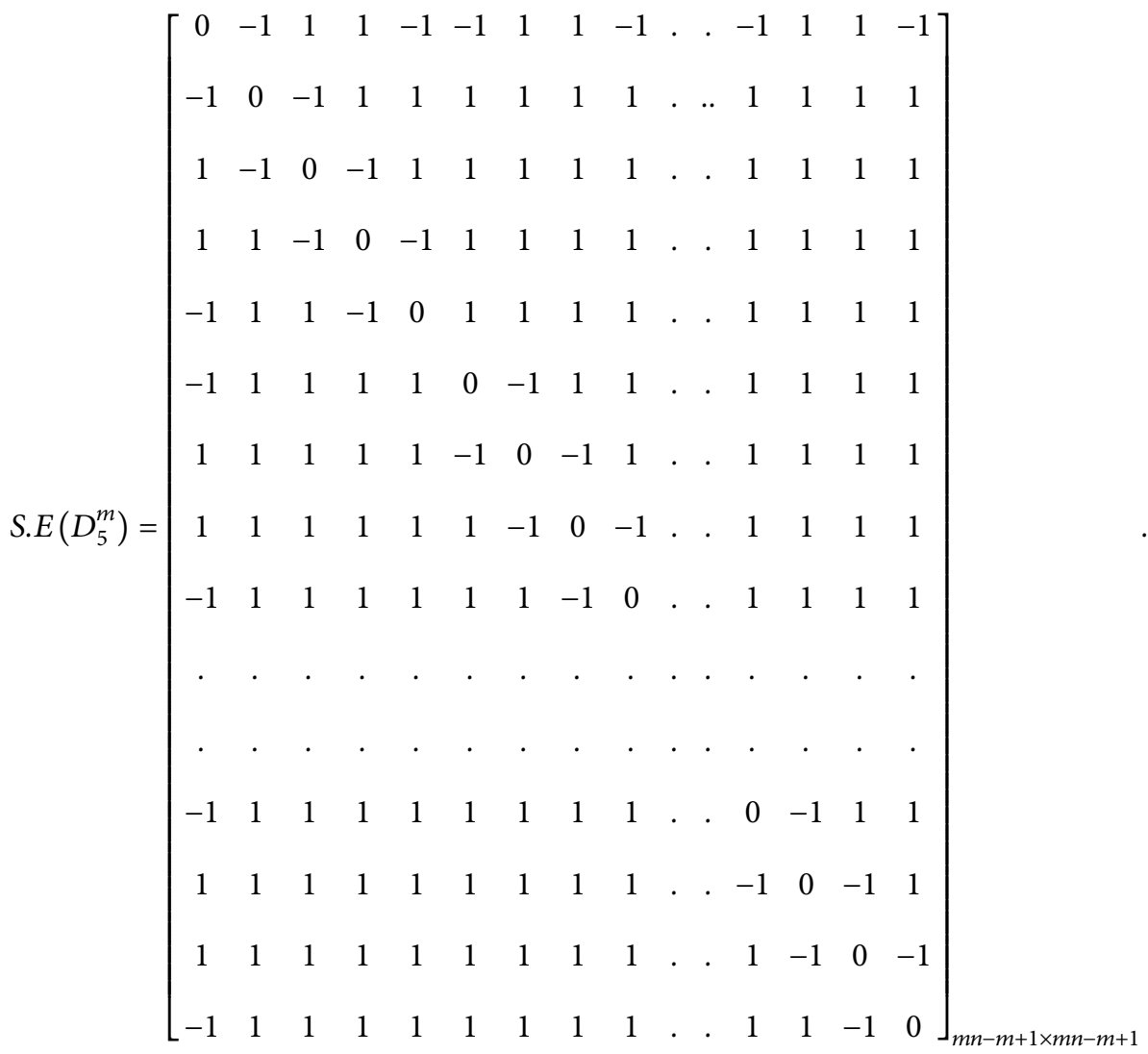

The characteristic equation of the above Seidel matrix is $\left(\lambda^{2}-5\right)^{m}\left(\lambda^{2}+4 \lambda-10\right)^{m-1}\left(\lambda^{3}-(4 m-4) \lambda^{2}-(4 m+1) \lambda+\right.$ $\left.16 m^{2}-16\right)=0$.

The eigenvalues of the above characteristic equation are $\lambda_{1}=\sqrt{5}(m)$ times, $\quad \lambda_{2}=-\sqrt{5}(m)$ times, $\lambda_{3}=-2+\sqrt{14}(m$ $-1)$ times, $\lambda_{4}=-2-\sqrt{14}(m-1)$ times, and $\lambda_{5}, \lambda_{6}, \lambda_{7}$, where $\lambda_{5}, \lambda_{6}, \lambda_{7}$ are the roots of the cubic equation given in the following:

$$
\left(\lambda^{3}-(4 m-4) \lambda^{2}-(4 m+1) \lambda+16 m^{2}-16\right)=0 .
$$

Now, Seidel energy can be calculated by using Definition 2.4.2 as follows:

$$
\begin{aligned}
\text { S.E. }\left(D_{5}^{m}\right) & =m\left|\lambda_{1}\right|+m\left|\lambda_{2}\right|+(m-1)\left|\lambda_{3}\right|+(m-1)\left|\lambda_{4}\right|+\left|\lambda_{5}\right|+\left|\lambda_{6}\right|+\left|\lambda_{7}\right| \\
& =m|\sqrt{5}|+m|-\sqrt{5}|+(m-1)|-2+\sqrt{14}|+(m-1)|-2-\sqrt{14}|+\left|\lambda_{5}\right|+\left|\lambda_{6}\right|+\left|\lambda_{7}\right| \\
& =m|\sqrt{5}|+m|-\sqrt{5}|+(m-1)|-2+\sqrt{14}|+(m-1)|-2-\sqrt{14}|+\chi \\
& =2[m(\sqrt{5}+\sqrt{14})-\sqrt{14}]+\chi, \chi=\left|\lambda_{5}\right|+\left|\lambda_{6}\right|+\left|\lambda_{7}\right| .
\end{aligned}
$$


Theorem 7. The Seidel energy of $D_{6}^{m}$ is $(5+4 \sqrt{3}) m \quad$ Proof. The Seidel matrix for $D_{6}^{m}$ is $-4 \sqrt{3}-1+\mathfrak{I}$.

$$
S\left(D_{6}^{m}\right)=\left[\begin{array}{cccccccccccccccccc}
0 & -1 & 1 & 1 & 1 & -1 & -1 & 1 & 1 & 1 & -1 & . & . & -1 & 1 & 1 & 1 & -1 \\
-1 & 0 & -1 & 1 & 1 & 1 & 1 & 1 & 1 & 1 & 1 & . & . & 1 & 1 & 1 & 1 & 1 \\
1 & -1 & 0 & -1 & 1 & 1 & 1 & 1 & 1 & 1 & 1 & . & . & 1 & 1 & 1 & 1 & 1 \\
1 & 1 & -1 & 0 & -1 & 1 & 1 & 1 & 1 & 1 & 1 & . & . & 1 & 1 & 1 & 1 & 1 \\
1 & 1 & 1 & -1 & 0 & -1 & 1 & 1 & 1 & 1 & 1 & . & . & 1 & 1 & 1 & 1 & 1 \\
-1 & 1 & 1 & 1 & -1 & 0 & 1 & 1 & 1 & 1 & 1 & . & . & 1 & 1 & 1 & 1 & 1 \\
-1 & 1 & 1 & 1 & 1 & 1 & 0 & -1 & 1 & 1 & 1 & . & . & 1 & 1 & 1 & 1 & 1 \\
1 & 1 & 1 & 1 & 1 & 1 & -1 & 0 & -1 & 1 & 1 & . & . & 1 & 1 & 1 & 1 & 1 \\
1 & 1 & 1 & 1 & 1 & 1 & 1 & -1 & 0 & -1 & 1 & . & . & 1 & 1 & 1 & 1 & 1 \\
-1 & 1 & 1 & 1 & 1 & 1 & 1 & 1 & 1 & -1 & 0 & . & . & 1 & 1 & 1 & 1 & 1 \\
. & . & . & . & . & . & . & . & . & . & . & . & . & . & . & . & . & . \\
. & . & . & . & . & . & . & . & . & . & . & . & . & . & . & . & . & . \\
-1 & 1 & 1 & 1 & 1 & 1 & 1 & 1 & 1 & 1 & 1 & . & . & 0 & -1 & 1 & 1 & 1 \\
1 & 1 & 1 & 1 & 1 & 1 & 1 & 1 & 1 & 1 & 1 & . & . & -1 & 0 & -1 & 1 & 1 \\
1 & 1 & 1 & 1 & 1 & 1 & 1 & 1 & 1 & 1 & 1 & . & . & 1 & -1 & 0 & -1 & 1 \\
1 & 1 & 1 & 1 & 1 & 1 & 1 & 1 & 1 & 1 & 1 & . & . & 1 & 1 & -1 & 0 & -1 \\
-1 & 1 & 1 & 1 & 1 & 1 & 1 & 1 & 1 & 1 & 1 & . & . & 1 & 1 & 1 & -1 & 0
\end{array}\right]_{m n-m+1 \times m n-m+1}
$$

The characteristic equation for the above Seidel matrix is $(\lambda+1)^{m-1}(\lambda-1)^{m}(\lambda+3)^{m}\left(\lambda^{2}+2 \lambda-11\right)^{m-1}\left(\lambda^{4}-(5 m-3)\right.$ $\lambda^{3}-(9-m) \lambda^{2}+\left(24 m^{2}+5 m-11\right) \lambda-m(40 m-31)=0$.

The eigenvalues of the above Seidel matrix are $\lambda_{1}=-1(m-1)$ times, $\quad \lambda_{2}=1$ ( $m$ times $), \quad \lambda_{3}=-1+2 \sqrt{3}$
( $m-1$ times), $\lambda_{4}=-1-2 \sqrt{3}(m-1$ times $)$, and $\lambda_{5}, \lambda_{6}, \lambda_{7}, \lambda_{8}$ where $\lambda_{5}, \lambda_{6}, \lambda_{7}, \lambda_{8}$ are roots of the biquadratic equation given in the following:

$$
\left(\lambda^{4}-(5 m-3) \lambda^{3}-(9-m) \lambda^{2}+\left(24 m^{2}+5 m-11\right) \lambda-m(40 m-31)=0 .\right.
$$

Now, Seidel energy can be calculated by using Definition 2.4.2 as follows:

$$
\begin{aligned}
& =(m-1)(1)+m(1)+m(3)+(m-1)(-1+2 \sqrt{3})+(m-1)(1+2 \sqrt{3})+\mathfrak{I} \\
& =m-1+m+3 m-m+1+2 \sqrt{3} m-2 \sqrt{3}+2 \sqrt{3} m-2 \sqrt{3}-1+m+\mathfrak{J} \\
& =5 m+4 \sqrt{3} m-4 \sqrt{3}-1+\mathfrak{I} \\
& =(5+4 \sqrt{3}) m-4 \sqrt{3}-1+\mathfrak{J}, \mathfrak{J}=\left|\lambda_{5}\right|+\left|\lambda_{6}\right|+\left|\lambda_{7}\right|+\left|\lambda_{8}\right| .
\end{aligned}
$$

Theorem 8. Let $G$ be a double-wheel graph; then, S.E $\left(W_{n}\right)>5+\sqrt{4 n^{2}-12 n+25}$.
Proof. A Seidel matrix of order $2 n+1$ for a double-wheel graph $W_{n}$ is 


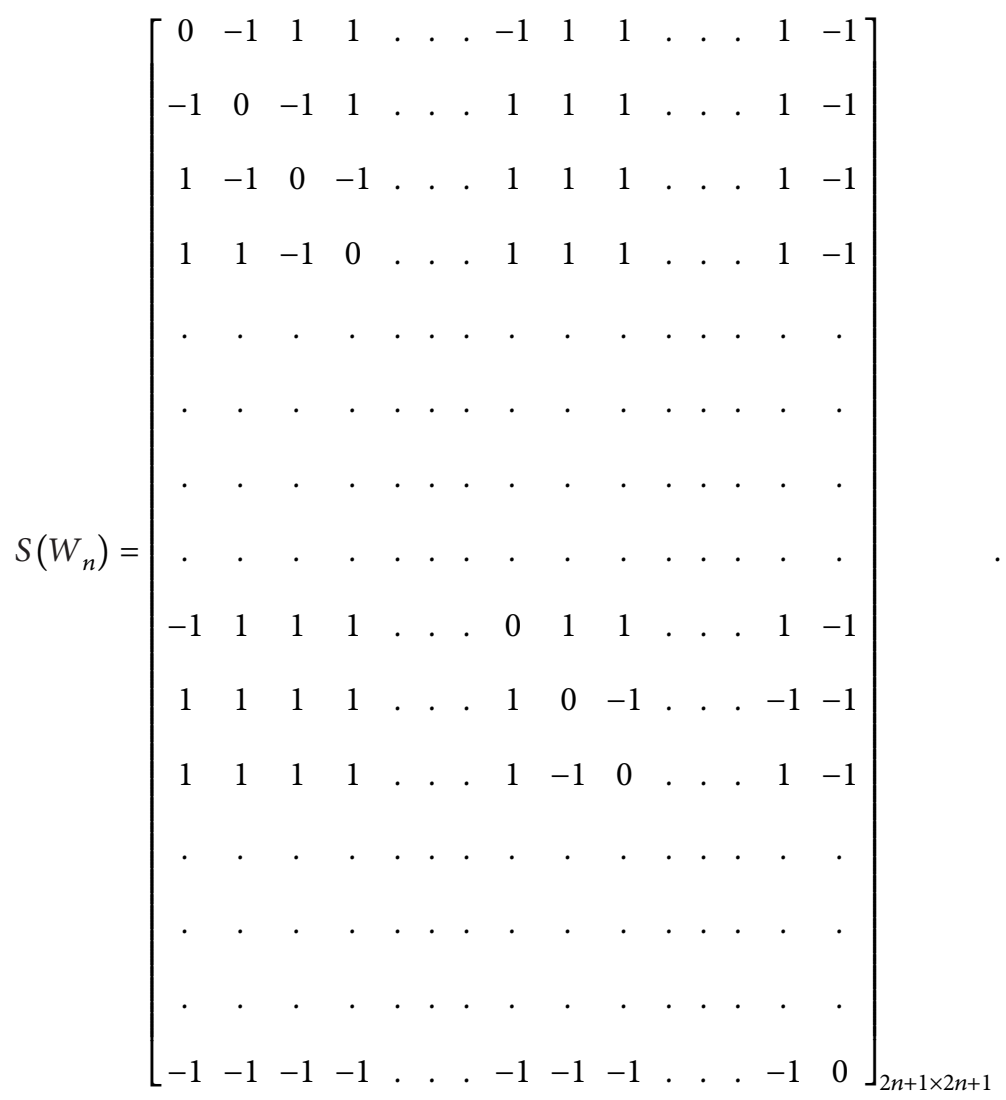

The characteristic equation of the Seidel matrix is $(\lambda+5)\left(\lambda^{2}-(2 n-5) \lambda-2 n\right)\left(\lambda^{2 n-2}+\lambda^{2 n-1}+\cdots \lambda\right)=0$.
The three roots of this characteristic equation are $\lambda_{1}=-5, \quad \lambda_{2}=\left((2 n-5)-\sqrt{4 n^{2}-12 n+25} / 2\right), \quad$ and $\lambda_{3}=\left((2 n-5)-\sqrt{4 n^{2}-12 n+25} / 2\right)$. Now, the Seidel energy can be calculated by using Definition 4 as follows:

$$
\begin{aligned}
\operatorname{S.E}\left(W_{n}\right)= & \left|\lambda_{1}\right|+\left|\lambda_{2}\right|+\left|\lambda_{3}\right|+\cdots\left|\lambda_{2 n+1}\right| \\
& >\left|\lambda_{1}\right|+\left|\lambda_{2}\right|+\left|\lambda_{3}\right| \\
= & |-5|+\left|\frac{(2 n-5)+\sqrt{4 n^{2}-12 n+25}}{2}\right|+\left|\frac{(2 n-5)-\sqrt{4 n^{2}-12 n+25}}{2}\right| \\
= & 5+\frac{(2 n-5)+\sqrt{4 n^{2}-12 n+25}}{2}+\frac{-2 n+5+\sqrt{4 n^{2}-12 n+25}}{2} \\
= & 5+\sqrt{4 n^{2}-12 n+25 .}
\end{aligned}
$$


Theorem 9. The Laplacian energy of Dutch windmill graph Proof. The Laplacian matrix for $D_{4}^{m}$ is given as follows: $D_{4}^{m}$ is $8 m$.

$$
L\left(D_{4}^{m}\right)=\left[\begin{array}{cccccccccccccccccc}
2 m & -1 & 0 & -1 & -1 & 0 & -1 & -1 & 0 & -1 & . & . & -1 & 0 & -1 & -1 & 0 & -1 \\
-1 & 2 & -1 & 0 & 0 & 0 & 0 & 0 & 0 & 0 & . & . & 0 & 0 & 0 & 0 & 0 & 0 \\
0 & -1 & 2 & -1 & 0 & 0 & 0 & 0 & 0 & 0 & . & . & 0 & 0 & 0 & 0 & 0 & 0 \\
-1 & 0 & -1 & 2 & 0 & 0 & 0 & 0 & 0 & 0 & . & . & 0 & 0 & 0 & 0 & 0 & 0 \\
-1 & 0 & 0 & 0 & 2 & -1 & 0 & 0 & 0 & 0 & . & . & 0 & 0 & 0 & 0 & 0 & 0 \\
0 & 0 & 0 & 0 & -1 & 2 & -1 & 0 & 0 & 0 & . & . & 0 & 0 & 0 & 0 & 0 & 0 \\
-1 & 0 & 0 & 0 & 0 & -1 & 2 & 0 & 0 & 0 & . & . & 0 & 0 & 0 & 0 & 0 & 0 \\
-1 & 0 & 0 & 0 & 0 & 0 & 0 & 2 & -1 & 0 & . & . & 0 & 0 & 0 & 0 & 0 & 0 \\
0 & 0 & 0 & 0 & 0 & 0 & 0 & -1 & 2 & -1 & . & . & 0 & 0 & 0 & 0 & 0 & 0 \\
-1 & 0 & 0 & 0 & 0 & 0 & 0 & 0 & -1 & 2 & . & . & 0 & 0 & 0 & 0 & 0 & 0 \\
. & . & . & . & . & . & . & . & . & . & . & . & . & . & . & . & . & . \\
. & . & . & . & . & . & . & . & . & . & . & . & . & . & . & . & . & . \\
-1 & 0 & 0 & 0 & 0 & 0 & 0 & 0 & 0 & 0 & . & . & 2 & -1 & 0 & 0 & 0 & 0 \\
0 & 0 & 0 & 0 & 0 & 0 & 0 & 0 & 0 & 0 & . & . & -1 & 2 & -1 & 0 & 0 & 0 \\
-1 & 0 & 0 & 0 & 0 & 0 & 0 & 0 & 0 & 0 & . & . & 0 & -1 & 2 & 0 & 0 & 0 \\
-1 & 0 & 0 & 0 & 0 & 0 & 0 & 0 & 0 & 0 & . & . & 0 & 0 & 0 & 2 & -1 & 0 \\
0 & 0 & 0 & 0 & 0 & 0 & 0 & 0 & 0 & 0 & . & . & 0 & 0 & 0 & -1 & 2 & -1 \\
-1 & 0 & 0 & 0 & 0 & 0 & 0 & 0 & 0 & 0 & . & . & 0 & 0 & 0 & 0 & -1 & 2
\end{array}\right]_{m n-m+1 \times m n-m+1}
$$

The characteristic equation of the above Laplacian matrix is given as follows:

$(\lambda-0)(\lambda-2)^{m}\left(\lambda^{2}-4 \lambda+2\right)^{m-1}\left(\lambda^{2}-(2 m+4) \lambda+6 m+2\right)=0$.
Eigenvalues are $\lambda_{1}=0, \lambda_{2}=2$ ( $m$ times $), \lambda_{3}=2+\sqrt{2}(m$ -1 times $), \quad \lambda_{4}=2-\sqrt{2}(m-1$ times $), \quad \lambda_{5}=m+2+$ $\sqrt{m^{2}-2 m+2}$, and $\lambda_{6}=m+2-\sqrt{m^{2}-2 m+2}$.

$$
\begin{aligned}
L . E(G)= & \left|m+2+\sqrt{m^{2}-2 m+2}\right|+\left|m+2-\sqrt{m^{2}-2 m+2}\right| \\
& +(m-1)|2+\sqrt{2}|+(m-1)|2-\sqrt{2}|+m|2| .
\end{aligned}
$$

So, Laplacian energy of Dutch windmill graph L.E $\left(D_{4}^{m}\right)=8 m$.
Theorem 10. The Laplacian energy of Dutch windmill graph $D_{5}^{m}$ is $10 \mathrm{~m}$. 
Proof. The Laplacian matrix for $D_{5}^{m}$ is given as follows:

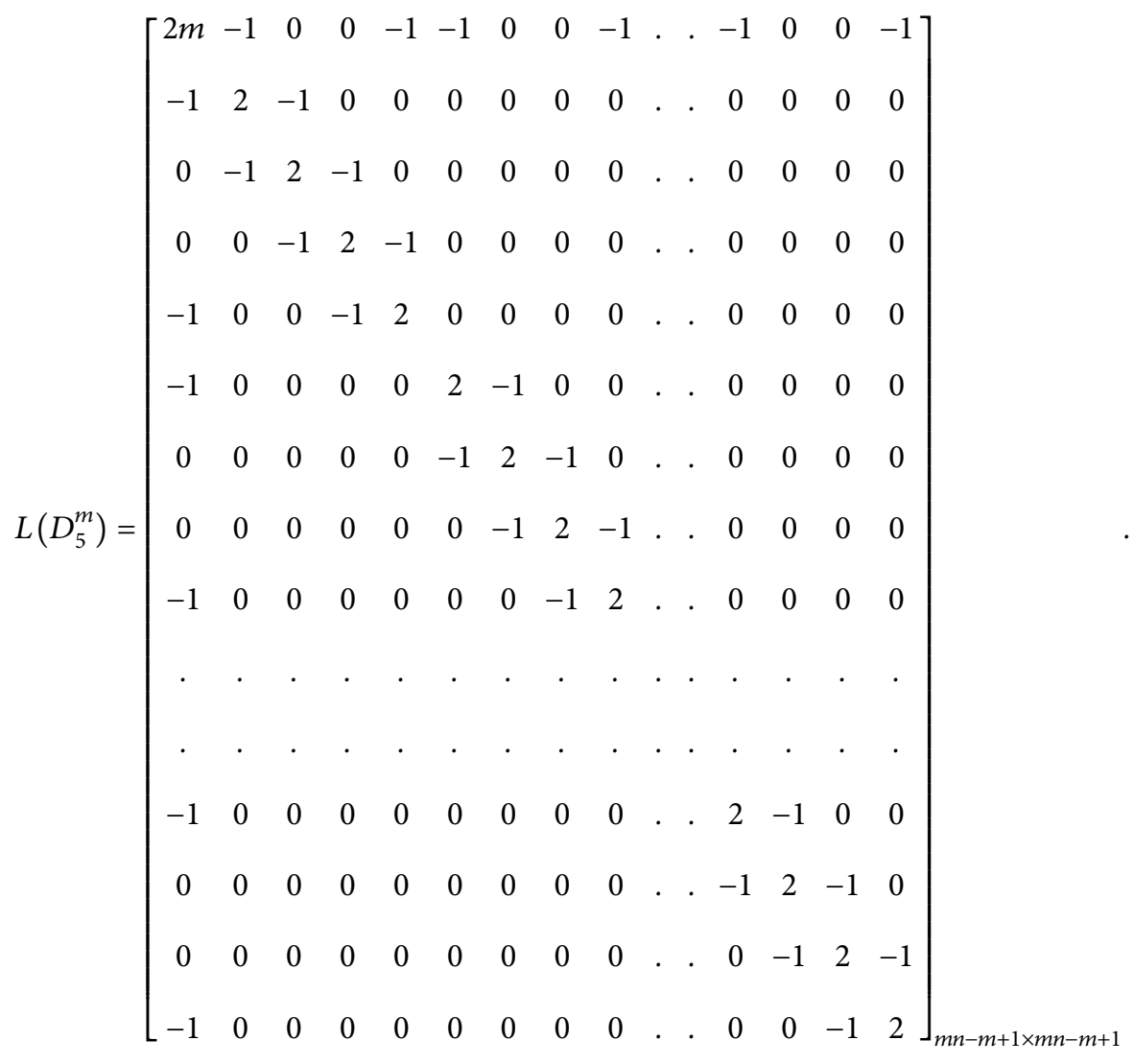

The characteristic equation of the above Laplacian matrix is given as follows:

$$
(\lambda-0)\left(\lambda^{2}-3 \lambda+1\right)^{m-1}\left(\lambda^{2}-5 \lambda+5\right)^{m}\left(\lambda^{2}-(2 m+3) \lambda+(4 m+1)\right)=0
$$

The eigenvalues of the above Laplacian matrix are given as follows. $\lambda_{1}=0, \lambda_{2}=(3+\sqrt{5} / 2)(m-1$ times $), \lambda_{3}=(3-$ $\sqrt{5} / 2)(m-1$ times $), \lambda_{4}=(5+\sqrt{5} / 2)(m$ times $), \lambda_{5}=(5-$ $\sqrt{5} / 2)(m$ times $), \lambda_{6}=\left((2 m+3)+\sqrt{4 m^{2}-4 m+5} / 2\right)$, and $\lambda_{7}=\left((2 m+3)-\sqrt{4 m^{2}-4 m+5} / 2\right)$.

So, the Laplacian energy is given by

$$
\begin{aligned}
& \operatorname{L.E}\left(D_{5}^{m}\right)=|0|+m\left|\frac{5+\sqrt{5}}{2}\right|+m\left|\frac{5-\sqrt{5}}{2}\right| \\
& +(m-1)\left|\frac{3+\sqrt{5}}{2}\right|+(m-1)\left|\frac{3-\sqrt{5}}{2}\right| \\
& +\left|\frac{(2 m+3)+\sqrt{4 m^{2}-4 m+5}}{2}\right|+\left|\frac{(2 m+3)-\sqrt{4 m^{2}-4 m+5}}{2}\right| \text {, } \\
& L . E\left(D_{5}^{m}\right)=10 m \text {. }
\end{aligned}
$$


Theorem 11. The Laplacian energy of the Dutch windmill graph is L.E $\left(D_{6}^{m}\right)=10 m-6+\chi$.
Proof. The Laplacian matrix for $D_{6}^{m}$ is given as follows:

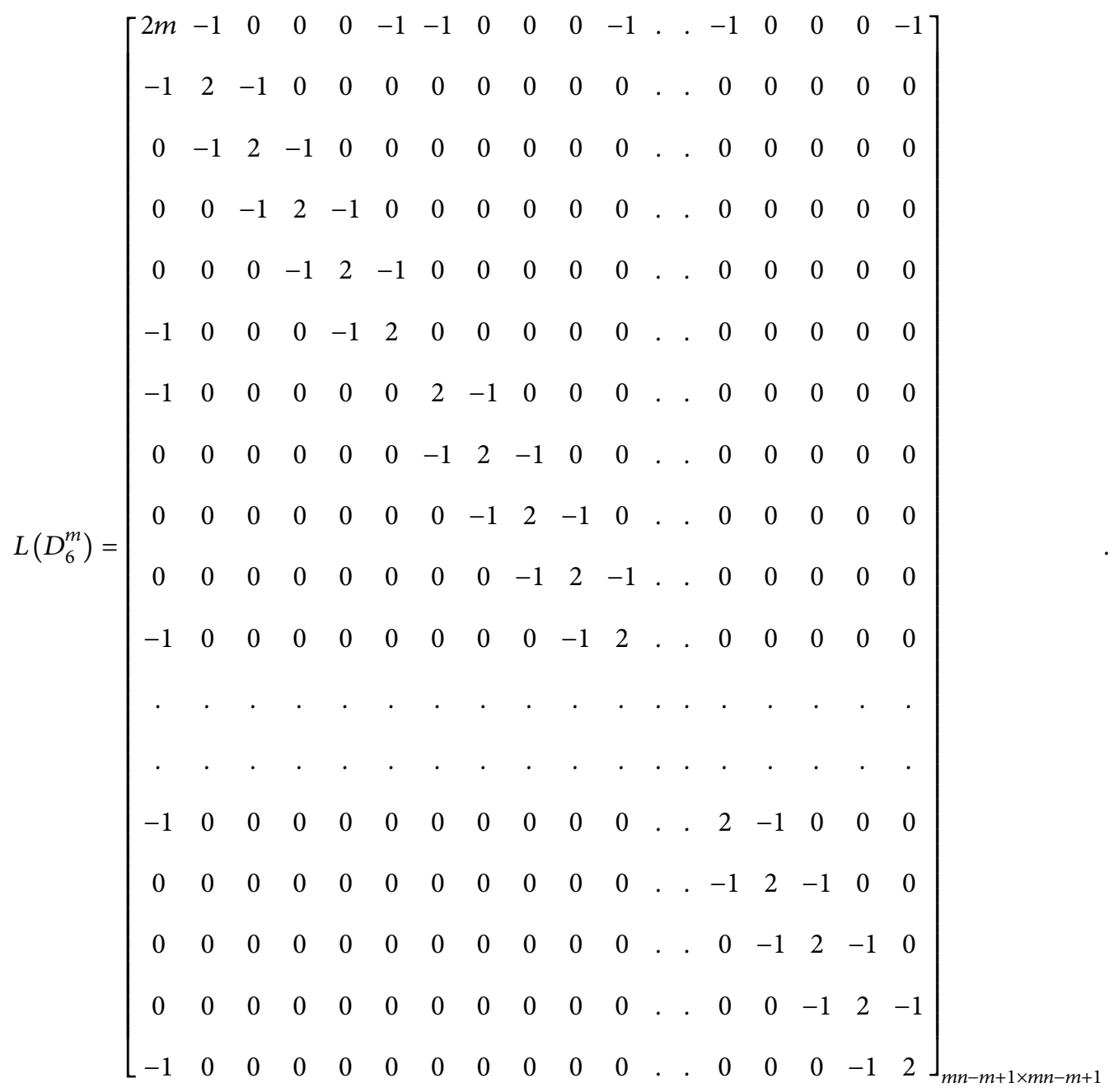

The characteristic equation of the above Laplacian matrix is given as follows:

$$
\begin{gathered}
(\lambda-0)(\lambda-1)^{m}(\lambda-2)^{m-1}(\lambda-3)^{m}\left(\lambda^{2}-4 \lambda+1\right)^{m-1} \\
\cdot\left(\lambda^{3}-(2 m+6) \lambda^{2}+(10 m+9) \lambda-(10 m+2)\right)=0 .
\end{gathered}
$$

The eigenvalues are $\lambda_{1}=0, \lambda_{2}=1$ ( $m$ times), $\lambda_{3}=2$ ( $m-1$ times), $\lambda_{4}=3$ ( $m$ times), $\lambda_{5}=2+\sqrt{3}(m-1$ times $)$, $\lambda_{6}=2-\sqrt{3}(m-1$ times $)$, and $\lambda_{7}, \lambda_{8}, \lambda_{9}$, where $\lambda_{7}, \lambda_{8}, \lambda_{9}$ are the roots of the cubic equation given in the following:

$$
\lambda^{3}-(2 m+6) \lambda^{2}+(10 m+9) \lambda-(10 m+2)=0 .
$$

Now, Laplacian energy $=\left|\lambda_{5}\right|+\left|\lambda_{6}\right|+\left|\lambda_{7}\right|+\left|\lambda_{8}\right|+\left|\lambda_{5}\right|+$ $\left|\lambda_{6}\right|+\left|\lambda_{7}\right|+\left|\lambda_{8}\right|+\left|\lambda_{9}\right|$.
So, the Laplacian energy of $D_{6}^{m}$ is given by

$$
\begin{gathered}
\operatorname{L.E}\left(D_{6}^{m}\right)=|0|+m|1|+(m-1)|2|+m|3|+(m-1) \mid 2 \\
+\sqrt{3}|+(m-1)| 2-\sqrt{3} \mid+\chi, \\
\text { where } \chi=\left|\lambda_{7}\right|+\left|\lambda_{8}\right|+\left|\lambda_{9}\right| . \\
\qquad . E\left(D_{6}^{m}\right)=10 m-6+\chi .
\end{gathered}
$$

Theorem 12. Let $G$ be a double-wheel graph $W_{n}$; then, L.E $(G)>2 n$.

Proof. A Laplacian matrix of order $2 n+1$ for a doublewheel graph $W_{n}, n \geq 3$, is given as follows: 


$$
L\left(W_{n}\right)=\left[\begin{array}{cccccccccccccc}
3 & -1 & 0 & 0 & . & . . & . & -1 & 0 & 0 & & 0 & -1 \\
-1 & 3 & -1 & 0 & . & . & . & 0 & 0 & 0 & & 0 & -1 \\
0 & -1 & 3 & -1 & . & . & . & 0 & 0 & 0 & . & . & 0 & -1 \\
0 & 0 & -1 & 3 & . & . & . & 0 & 0 & 0 & . & . & 0 & -1 \\
. & . & . & . & . & . & . & . & . & . & . & . & . & . \\
. & . & . & . & . & . & . & . & . & . & . & . & . & . \\
-1 & 0 & 0 & 0 & . & . & . & 3 & 0 & 0 & . & . & 0 & -1 \\
0 & 0 & 0 & 0 & . & . & . & 0 & 3 & -1 & . & . . & -1 & -1 \\
0 & 0 & 0 & 0 & . & . & . & 0 & -1 & 3 & . & . & 0 & -1 \\
. & . & . & . & . . & . & . . & . & . & . & . & . & . & . \\
. & . & . & . & . & . & . & . & . & . & . & . & . & . \\
0 & 0 & 0 & 0 & . & . & . & 0 & -1 & 0 & . . & . & 3 & -1 \\
-1 & -1 & -1 & -1 & . & . & . & -1 & -1 & -1 & . & . & -1 & 2 n
\end{array}\right]_{2 n+1 \times 2 n+1}
$$

The characteristic equation for the Laplacian matrix is given as follows:

$$
\begin{aligned}
\operatorname{det}(A-\lambda I) & =0, \\
(\lambda-0)\left(\lambda-(2 n-1)(\lambda-1)\left(\lambda^{2 n-2}+\lambda^{2 n-1}+\cdots \lambda\right)\right) & =0 .
\end{aligned}
$$

The roots of this characteristic equation are $\lambda_{1}, \lambda_{2}$, $\lambda_{3}, \ldots, \lambda_{2 n+1}$. The three roots of the above characteristic equation are $\lambda_{1}=0, \lambda_{2}=1$, and $\lambda_{3}=2 n-1$. The Laplacian energy is defined as

$$
\text { L.E. }(G)=\left|\lambda_{1}\right|+\left|\lambda_{2}\right|+\left|\lambda_{3}\right|+\cdots+\left|\lambda_{2 n+1}\right| .
$$
$\left|\lambda_{3}\right|$

Clearly, $\quad\left|\lambda_{1}\right|+\left|\lambda_{2}\right|+\left|\lambda_{3}\right|+\cdots \cdots+\left|\lambda_{2 n+1}\right|>\left|\lambda_{1}\right|+\left|\lambda_{2}\right|+$

$$
=2 n \text {. }
$$

So, $L . E(G)>2 n$.

Theorem 13. The color energy for $D_{4}^{m}$ is given by $(1+2$ $\sqrt{2}) m-2 \sqrt{2}+\chi$.

Proof. The color matrix for $D_{4}^{m}$ is given as follows:

$$
C\left(D_{4}^{m}\right)=\left[\begin{array}{cccccccccccc}
0 & 1 & -1 & 1 & 1 & -1 & 1 & . & . & 1 & -1 & 1 \\
1 & 0 & 1 & -1 & -1 & 0 & -1 & . & . & -1 & 0 & -1 \\
-1 & 1 & 0 & 1 & 0 & -1 & 0 & . & . & 0 & -1 & 0 \\
1 & -1 & 1 & 0 & -1 & 0 & -1 & . & . & -1 & 0 & -1 \\
1 & -1 & 0 & -1 & 0 & 1 & -1 & . & . & -1 & 0 & -1 \\
-1 & 0 & -1 & 0 & 1 & 0 & 1 & . & . & 0 & -1 & 0 \\
1 & -1 & 0 & -1 & -1 & 1 & 0 & . & . & -1 & 0 & -1 \\
\cdot & . & . & . & . & . & . & . & . & . & . & . \\
. & . & . & . & . & . & . & . & . & . & . & . \\
1 & -1 & 0 & -1 & -1 & 0 & -1 & . & . & 0 & 1 & -1 \\
-1 & 0 & -1 & 0 & 0 & -1 & 0 & . & . & 1 & 0 & 1 \\
1 & -1 & 0 & -1 & -1 & 0 & -1 & . & . & -1 & 1 & 0
\end{array}\right]_{m n-m+1 \times m n-m+1}
$$


The characteristic equation of the above color matrix is given as follows:

$$
\begin{gathered}
(\lambda-1)^{m}\left(\lambda^{2}-2 \lambda-1\right)^{m-1}\left(\lambda^{3}+(3 m-2) \lambda^{2}\right. \\
+\left(2 m^{2}-6 m-1\right) \lambda-\left(4 m^{2}-7 m\right)=0 .
\end{gathered}
$$

$\lambda_{1}=1$ ( $m$ times $), \quad \lambda_{2}=1+\sqrt{2}(m-1$ times $), \quad \lambda_{3}=1-$ $\sqrt{2}(m-1$ times $)$, and $\lambda_{4}, \lambda_{5}, \lambda_{6}$ are the eigenvalues of the above characteristic equation, where $\lambda_{4}, \lambda_{5}, \lambda_{6}$ are the roots of the cubic equation given in the following:

$$
\left(\lambda^{3}+(3 m-2) \lambda^{2}+\left(2 m^{2}-6 m-1\right) \lambda-\left(4 m^{2}-7 m\right)=0 .\right.
$$

So, the color energy of

$$
\begin{aligned}
D_{4}^{m}= & m|1|+(m-1)|1+\sqrt{2}|+(m-1)|1-\sqrt{2}| \\
& +\left|\lambda_{4}\right|+\left|\lambda_{5}\right|+\left|\lambda_{6}\right|, \\
= & m|1|+(m-1)|1+\sqrt{2}|+(m-1)|1-\sqrt{2}|+\chi,
\end{aligned}
$$

where $\chi=\left|\lambda_{4}\right|+\left|\lambda_{5}\right|+\left|\lambda_{6}\right|$.

$$
\text { So, } C . E\left(D_{4}^{m}\right)=(1+2 \sqrt{2}) m-2 \sqrt{2}+\chi \text {. }
$$

Theorem 14. The color energy of $D_{5}^{m}$ is given by $(m-1)(3+\sqrt{5})+\mathfrak{\Im}$.

Proof. The color matrix for $D_{5}^{m}$ of order $m n-m+1$ is given as follows:

$$
C\left(D_{5}^{m}\right)=\left[\begin{array}{cccccccccccccccc}
0 & 1 & -1 & 0 & 1 & 1 & -1 & 0 & 1 & . & . & . & 1 & -1 & 0 & 1 \\
1 & 0 & 0 & -1 & 0 & -1 & 0 & -1 & 0 & . & . & . & -1 & 0 & -1 & 0 \\
-1 & 1 & 1 & 1 & 0 & 0 & -1 & 0 & 0 & . & . & . & 0 & -1 & 0 & 0 \\
0 & -1 & 1 & 0 & 1 & -1 & 0 & -1 & 0 & . & . & . & -1 & 0 & -1 & 0 \\
1 & 0 & 0 & 1 & 0 & 0 & 0 & 0 & -1 & . & . & . & 0 & 0 & 0 & -1 \\
1 & -1 & 0 & -1 & 0 & 0 & 1 & -1 & 0 & . & . & . & -1 & 0 & -1 & 0 \\
-1 & 0 & -1 & 0 & 0 & 1 & 0 & 1 & 0 & . & . & . & 0 & -1 & 0 & 0 \\
0 & -1 & 0 & -1 & 0 & -1 & 1 & 0 & 1 & . & . & . & -1 & 0 & -1 & 0 \\
1 & 0 & 0 & 0 & -1 & 0 & 0 & 1 & 0 & . & . & . & 0 & 0 & 0 & -1 \\
. & . & . & . & . & . & . & . & . & . & . & . & . & . & . & . \\
. & . & . & . & . & . & . & . & . & . & . & . & . & . & . & . \\
. & . & . & . & . & . & . & . & . & . & . & . & . & . & . & . \\
1 & -1 & 0 & -1 & 0 & -1 & 0 & -1 & 0 & . & . & . & 0 & 1 & -1 & 0 \\
-1 & 0 & -1 & 0 & 0 & 0 & -1 & 0 & 0 & . & . & . & 1 & 0 & 1 & 0 \\
0 & -1 & 0 & -1 & 0 & -1 & 0 & -1 & 0 & . & . & . & -1 & 1 & 0 & 1 \\
1 & 0 & 0 & 0 & -1 & 0 & 0 & 0 & -1 & . & . & . & 0 & 0 & 1 & 0
\end{array}\right]_{m n-m+1 \times m n-m+1}
$$

The characteristic equation is given as follows:

$$
\begin{aligned}
& \left(\lambda^{2}-\lambda-1\right)^{m-1}\left(\lambda^{2}-3 \lambda+1\right)^{m-1}\left(\lambda^{5}+4(m-1) \lambda^{4}\right. \\
& +\left(5 m^{2}-15 m+3\right) \lambda^{3}+\left(-2 m^{3}+19 m^{2}-19 m-2\right) \lambda^{2} \\
& +\left(-9 m^{3}+26 m^{2}-6 m-1\right) \lambda+m\left(-m^{3}+9 m^{2}-10 m-6\right)=0 .
\end{aligned}
$$

The eigenvalues are $\lambda_{1}=1+\sqrt{5} / 2(m-1$ times $), \lambda_{2}=1-$ $\sqrt{5} / 2$ ( $m-1$ times), $\quad \lambda_{3}=3+\sqrt{5} / 2$ ( $m-1$ times $), \quad \lambda_{4}=3$ $-\sqrt{5} / 2\left(m-1\right.$ times), and $\lambda_{5}, \lambda_{6}, \lambda_{7}, \lambda_{8}, \lambda_{9}$. Here, $\lambda_{5}, \lambda_{6}, \lambda_{7}$, $\lambda_{8}, \lambda_{9}$ are the roots of the equation given in the following:

$$
\begin{aligned}
& \left(\lambda^{5}+4(m-1) \lambda^{4}+\left(5 m^{2}-15 m+3\right) \lambda^{3}+\left(-2 m^{3}+19 m^{2}-19 m-2\right) \lambda^{2}\right. \\
& +\left(-9 m^{3}+26 m^{2}-6 m-1\right) \lambda+m\left(-m^{3}+9 m^{2}-10 m-6\right)=0
\end{aligned}
$$


Now, color energy $=\left|\lambda_{1}\right|+\left|\lambda_{2}\right|+\left|\lambda_{3}\right|+\left|\lambda_{4}\right|+\left|\lambda_{5}\right|+\left|\lambda_{6}\right|+$ $\left|\lambda_{7}\right|+\left|\lambda_{8}\right|+\left|\lambda_{9}\right|$.

$$
\begin{aligned}
& \text { C.E }\left(D_{5}^{m}\right)=(m-1)\left|\frac{1+\sqrt{5}}{2}\right|+(m-1)\left|\frac{1-\sqrt{5}}{2}\right|+(m-1)\left|\frac{3-\sqrt{5}}{2}\right|+(m-1)\left|\frac{3+\sqrt{5}}{2}\right|+\left|\lambda_{5}\right|+\left|\lambda_{6}\right|+\left|\lambda_{7}\right|+\left|\lambda_{8}\right|+\left|\lambda_{9}\right|, \\
& C . E\left(D_{5}^{m}\right)=(m-1)\left|\frac{1+\sqrt{5}}{2}\right|+(m-1)\left|\frac{1-\sqrt{5}}{2}\right|+(m-1)\left|\frac{3-\sqrt{5}}{2}\right|+(m-1)\left|\frac{3+\sqrt{5}}{2}\right|+\mathfrak{J},
\end{aligned}
$$

where $\mathfrak{\Im}=\left|\lambda_{5}\right|+\left|\lambda_{6}\right|+\left|\lambda_{7}\right|+\left|\lambda_{8}\right|+\left|\lambda_{9}\right|$.

So, C.E $\left(D_{5}^{m}\right)=(m-1)(3+\sqrt{5})+\mathfrak{I}$.
Theorem 15. The color energy for $D_{6}^{m}$ is given by $m(3+2 \sqrt{3})-2 \sqrt{3}-1+\mathfrak{\Im}$.

Proof. The color matrix for $D_{6}^{m}$ is given as follows:

$$
C\left(D_{6}^{m}\right)=\left[\begin{array}{cccccccccccccccccc}
0 & 1 & -1 & 0 & -1 & 1 & 1 & -1 & 0 & -1 & 1 & . & . & 1 & -1 & 0 & -1 & 1 \\
1 & 0 & 1 & -1 & 0 & -1 & -1 & 0 & -1 & 0 & -1 & . & . & -1 & 0 & -1 & 0 & -1 \\
-1 & 1 & 0 & 1 & -1 & 0 & 0 & -1 & 0 & -1 & 0 & . & . & 0 & -1 & 0 & -1 & 0 \\
0 & -1 & 1 & 0 & 1 & -1 & -1 & 0 & -1 & 0 & -1 & . & . & -1 & 0 & -1 & 0 & -1 \\
-1 & 0 & -1 & 1 & 0 & 1 & 0 & -1 & 0 & -1 & 0 & . & . & 0 & -1 & 0 & -1 & 0 \\
1 & -1 & 0 & -1 & 1 & 0 & -1 & 0 & -1 & 0 & -1 & . & . & -1 & 0 & -1 & 0 & -1 \\
1 & -1 & 0 & -1 & 0 & -1 & 0 & 1 & -1 & 0 & -1 & . & . & -1 & 0 & -1 & 0 & -1 \\
-1 & 0 & -1 & 0 & -1 & 0 & 1 & 0 & 1 & -1 & 0 & . & . & 0 & -1 & 0 & -1 & 0 \\
0 & -1 & 0 & -1 & 0 & -1 & -1 & 1 & 0 & 1 & -1 & . & . & -1 & 0 & -1 & 0 & -1 \\
-1 & 0 & -1 & 0 & -1 & 0 & 0 & -1 & 1 & 0 & 1 & . & . & 0 & -1 & 0 & -1 & 0 \\
1 & -1 & 0 & -1 & 0 & -1 & -1 & 0 & -1 & 1 & 0 & . & . & -1 & 0 & -1 & 0 & -1 \\
& . & . & . & . & . & . & . & . & . & . & . & . & . & . & . & . & . \\
& . & . & . & . & . & . & . & . & . & . & . & . & . & . & . & . & . \\
1 & -1 & 0 & -1 & 0 & -1 & -1 & 0 & -1 & 0 & -1 & . & . & 0 & 1 & -1 & 0 & -1 \\
-1 & 0 & -1 & 0 & -1 & 0 & 0 & -1 & 0 & -1 & 0 & . & . & 1 & 0 & 1 & -1 & 0 \\
0 & -1 & 0 & -1 & 0 & -1 & -1 & 0 & -1 & 0 & -1 & . & . & -1 & 1 & 0 & 1 & -1 \\
-1 & 0 & -1 & 0 & -1 & 0 & 0 & -1 & 0 & -1 & 0 & . & . & 0 & -1 & 1 & 0 & 1 \\
1 & -1 & 0 & -1 & 0 & -1 & -1 & 0 & -1 & 0 & -1 & . & . & -1 & 0 & -1 & 1 & 0
\end{array}\right]_{m n-m+1 \times m n-m+1}
$$

The characteristic equation for the above color matrix is given as follows:

$$
(\lambda-0)^{m}(\lambda-1)^{m-1}(\lambda-2)^{m}\left(\lambda^{2}-2 \lambda-2\right)^{m-1}\left(\lambda^{4}+(5 m-3) \lambda^{3}+\left(6 m^{2}-14 m\right) \lambda^{2}-\left(18 m^{2}-16 m-2\right) \lambda-(2 m-2)^{2} m\right)=0
$$

The eigenvalues are $\lambda_{1}=0(m)$ times, $\lambda_{2}=1(m-1)$ times, $\lambda_{3}=2(m)$ times, $\lambda_{4}=1+\sqrt{3}(m-1)$ times, $\lambda_{5}=1-$ $\sqrt{3}(m-1)$ times, and $\lambda_{6}, \lambda_{7}, \lambda_{8}, \lambda_{9}$, where $\lambda_{6}, \lambda_{7}, \lambda_{8}, \lambda_{9}$ are the roots of the biquadratic equation given in the following: 


$$
\left(\lambda^{4}+(5 m-3) \lambda^{3}+\left(6 m^{2}-14 m\right) \lambda^{2}-\left(18 m^{2}-16 m-2\right) \lambda-(2 m-2)^{2} m\right)=0 .
$$

Now, color energy is

$$
\begin{aligned}
& \text { C.E }\left(D_{6}^{m}\right)=\left|\lambda_{1}\right|+\left|\lambda_{2}\right|+\left|\lambda_{3}\right|+\left|\lambda_{4}\right|+\left|\lambda_{5}\right|+\left|\lambda_{6}\right|+\left|\lambda_{7}\right|+\left|\lambda_{8}\right|+\left|\lambda_{9}\right|, \\
& \text { C.E }\left(D_{6}^{m}\right)=m|0|+(m-1)|1|+m|2|+(m-1)|1+\sqrt{3}|+(m-1)|1-\sqrt{3}|+\left|\lambda_{6}\right|+\left|\lambda_{7}\right|+\left|\lambda_{8}\right|+\left|\lambda_{9}\right|, \\
& \text { C.E }\left(D_{6}^{m}\right)=m|0|+(m-1)|1|+m|2|+(m-1)|1+\sqrt{3}|+(m-1)|1-\sqrt{3}|+\mathfrak{J},
\end{aligned}
$$

where $\mathfrak{J}=\left|\lambda_{6}\right|+\left|\lambda_{7}\right|+\left|\lambda_{8}\right|+\left|\lambda_{9}\right|$.

So, $C . E\left(D_{6}^{m}\right)=m(3+2 \sqrt{3})-2 \sqrt{3}-1+\mathfrak{I}$.

Proof. The color matrix for a double-wheel graph is given as follows:

Theorem 16. Let $G$ be a double-wheel graph; then, C.E $\left(W_{n}\right)>3+\sqrt{1+8 n}$.

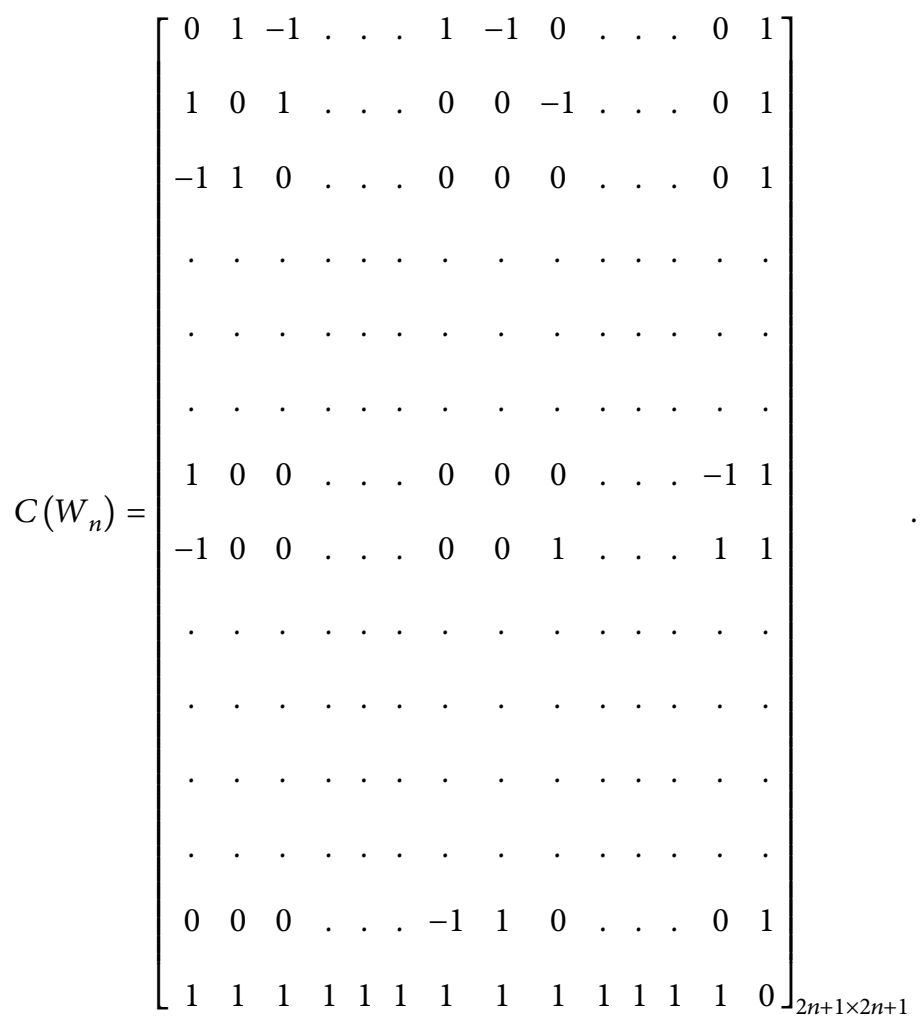

The characteristic equation of the color matrix is given as follows:

$$
\begin{aligned}
\operatorname{det}(A-\lambda I) & =0, \\
(\lambda-3)\left(\lambda^{2}-\lambda-2 n\right)\left(\lambda^{2 n-2}+\lambda^{2 n-1}+\cdots \lambda\right) & =0 .
\end{aligned}
$$

The roots of this characteristic equation are $\lambda_{1}, \lambda_{2}, \lambda_{3}$, $\ldots, \lambda_{n}$. The three roots of this characteristic equation are $\lambda_{1}=3, \lambda_{2}=(1+\sqrt{1+8 n} / 2)$, and $\lambda_{3}=(1-\sqrt{1+8 n} / 2)$.

Clearly, $\left|\lambda_{1}\right|+\left|\lambda_{2}\right|+\left|\lambda_{3}\right|+\cdots+\left|\lambda_{2 n+1}\right|>\left|\lambda_{1}\right|+\left|\lambda_{2}\right|+\left|\lambda_{3}\right|$

$$
\begin{aligned}
& =3+\frac{1+\sqrt{1+8 n}}{2}+\frac{-1+\sqrt{1+8 n}}{2}, \\
& =3+\sqrt{1+8 n} .
\end{aligned}
$$

C.E $\left(W_{n}\right)=\left|\lambda_{1}\right|+\left|\lambda_{2}\right|+\left|\lambda_{3}\right|+\cdots\left|\lambda_{n}\right|$. So, C.E $\left(W_{n}\right)>3+\sqrt{1+8 n}$.

Theorem 17. The distance energy of Dutch windmill graph $D_{4}^{m}$ is $2\left[(4 m-3)+3 \sqrt{2 m^{2}-2 m+1}\right]$. 
Proof. The distance matrix for Dutch windmill graph $D_{4}^{m}$ is

$$
D\left(D_{4}^{m}\right)=\left[\begin{array}{lllllll}
0 & 1 & 2 & 1 & 1 & 2 & 1 \\
1 & 0 & 1 & 2 & 2 & 3 & 2 \\
2 & 1 & 0 & 1 & 3 & 4 & 3 \\
1 & 2 & 1 & 0 & 2 & 3 & 2 \\
1 & 2 & 3 & 2 & 0 & 1 & 2 \\
2 & 3 & 4 & 3 & 1 & 0 & 1 \\
1 & 2 & 3 & 2 & 2 & 1 & 0 \\
& & & & & & \\
& & & & & & \\
1 & 2 & 3 & 2 & 2 & 3 & 2 \\
2 & 3 & 4 & 3 & 3 & 4 & 3 \\
1 & 2 & 3 & 2 & 2 & 3 & 2
\end{array}\right.
$$

The characteristic equation is $(\lambda+6)^{m-1}(\lambda+2)^{m}(\lambda-$ $0)^{m}\left(\lambda^{2}-(8 m-6) \lambda-\left(2 m^{2}+6 m\right)\right)=0$.

The eigenvalues are $\lambda_{1}=-6(m-1$ times $), \lambda_{2}=-2(m$ times), $\lambda_{3}=0$ ( $m$ times), $\lambda_{4}=(4 m-3)+3 \sqrt{2 m^{2}-2 m+1}$, and $\lambda_{5}=(4 m-3)-3 \sqrt{2 m^{2}-2 m+1}$.

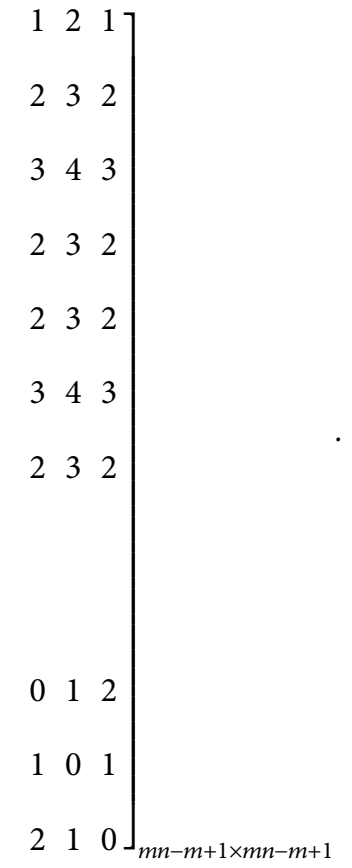

(67)

The distance energy can be calculated by using Definition 2.4 .3 as follows:

$$
\begin{aligned}
\operatorname{D.E.}\left(D_{4}^{m}\right)= & (m-1)\left|\lambda_{1}\right|+m\left|\lambda_{2}\right|+m\left|\lambda_{3}\right|+\left|\lambda_{4}\right|+\left|\lambda_{5}\right| \\
= & (m-1)|-6|+m|-2|+m|0|+\left|(4 m-3)+3 \sqrt{2 m^{2}-2 m+1}\right| \\
& +\left|(4 m-3)-3 \sqrt{2 m^{2}-2 m+1}\right| \\
= & 6 m-6+2 m+4 m-3+3 \sqrt{2 m^{2}-2 m+1}-4 m+3+3 \sqrt{2 m^{2}-2 m+1} \\
= & 8 m+6 \sqrt{2 m^{2}-2 m+1}-6 \\
= & 2\left(4 m-3+3 \sqrt{2 m^{2}-2 m+1}\right) .
\end{aligned}
$$

Theorem 18. The distance energy of $D_{5}^{m}$ is $12 m-9+\mathfrak{I}$, where $\mathfrak{I}$ is the sum of the absolute values of the roots of the $=0$. 
Proof. The distance matrix for $D_{5}^{m}$ is

$$
D\left(D_{5}^{m}\right)=\left[\begin{array}{lllllllllllllll}
0 & 1 & 2 & 2 & 1 & 1 & 2 & 2 & 1 & . & . & 1 & 2 & 2 & 1 \\
1 & 0 & 1 & 2 & 2 & 2 & 3 & 3 & 2 & . & . & 2 & 3 & 3 & 2 \\
2 & 1 & 0 & 1 & 2 & 3 & 4 & 4 & 3 & . & . & 3 & 4 & 4 & 3 \\
2 & 2 & 1 & 0 & 1 & 3 & 4 & 4 & 3 & . & . & 3 & 4 & 4 & 3 \\
1 & 2 & 2 & 1 & 0 & 2 & 3 & 3 & 2 & . & . & 2 & 3 & 3 & 2 \\
1 & 2 & 3 & 3 & 2 & 0 & 1 & 2 & 2 & . & . & 2 & 3 & 3 & 2 \\
2 & 3 & 4 & 4 & 3 & 1 & 0 & 1 & 2 & . & . & 3 & 4 & 4 & 3 \\
2 & 3 & 4 & 4 & 3 & 2 & 1 & 0 & 1 & . & . & 3 & 4 & 4 & 3 \\
1 & 2 & 3 & 3 & 2 & 2 & 2 & 1 & 0 & . & . & 2 & 3 & 3 & 2 \\
. & . & . & . & . & . & . & . & . & . & . & . & . & . & . \\
. & . & . & . & . & . & . & . & . & . & . & . & . & . & . \\
1 & 2 & 3 & 3 & 2 & 2 & 3 & 3 & 2 & . & . & 0 & 1 & 2 & 2 \\
2 & 3 & 4 & 4 & 3 & 3 & 4 & 4 & 3 & . & . & 1 & 0 & 1 & 2 \\
2 & 3 & 4 & 4 & 3 & 3 & 4 & 4 & 3 & . & . & 2 & 1 & 0 & 1 \\
1 & 2 & 3 & 3 & 2 & 2 & 3 & 3 & 2 & . & . & 2 & 2 & 1 & 0
\end{array}\right]_{m n-m+1 \times m n-m+1}
$$
is

The characteristic equation of the above distance matrix

$$
\begin{aligned}
&\left(\lambda^{2}+9 \lambda+5\right)^{m-1}\left(\lambda^{2}+3 \lambda+1\right)^{m}\left(\lambda^{3}-(12 m-9) \lambda^{2}-\left(4(m+3)^{2}-6 m-41\right) \lambda-6 m\right)=0 \\
& \lambda^{3}-(12 m-9) \lambda^{2}-\left(4(m+3)^{2}-6 m-41\right) \lambda-6 m=0
\end{aligned}
$$

The eigenvalues of the above characteristic equation are $\lambda_{1}, \lambda_{2}, \lambda_{3}, \quad \lambda_{4}=(-9+\sqrt{61} / 2)(m-1$ times $), \quad \lambda_{5}=(-9-$ $\sqrt{61} / 2)(m-1$ times $), \quad \lambda_{6}=(-3+\sqrt{5} / 2)(m$ times $), \quad$ and $\lambda_{7}=(-3-\sqrt{5} / 2)$ ( $m$ times), where $\lambda_{1}, \lambda_{2}, \lambda_{3}$ are the roots of the cubic equation given in the following:
Now, the distance energy of $D_{5}^{m}$ can be calculated by using Definition 2.4.3 as follows:

$$
\begin{aligned}
\text { D.E. }\left(D_{5}^{m}\right) & =\left|\lambda_{1}\right|+\left|\lambda_{2}\right|+\left|\lambda_{3}\right|+(m-1)\left|\lambda_{4}\right|+(m-1)\left|\lambda_{5}\right|+m\left|\lambda_{6}\right|+m\left|\lambda_{7}\right| \\
& =\left|\lambda_{1}\right|+\left|\lambda_{2}\right|+\left|\lambda_{3}\right|+(m-1)\left|\frac{-9+\sqrt{61}}{2}\right|+(m-1)\left|\frac{-9-\sqrt{61}}{2}\right|+m\left|\frac{-3+\sqrt{5}}{2}\right|+m\left|\frac{-3-\sqrt{5}}{2}\right| \\
& =\left|\lambda_{1}\right|+\left|\lambda_{2}\right|+\left|\lambda_{3}\right|+(m-1)\left(\frac{9-\sqrt{61}}{2}\right)+(m-1)\left(\frac{9+\sqrt{61}}{2}\right)+m\left(\frac{3-\sqrt{5}}{2}\right)+m\left(\frac{3+\sqrt{5}}{2}\right) \\
& =\left|\lambda_{1}\right|+\left|\lambda_{2}\right|+\left|\lambda_{3}\right|+\frac{9 m-9-\sqrt{61} m+\sqrt{61}+9 m-9+\sqrt{61} m-\sqrt{61}+3 m-\sqrt{5} m+3 m+\sqrt{5} m}{2} \\
& =\left|\lambda_{1}\right|+\left|\lambda_{2}\right|+\left|\lambda_{3}\right|+12 m-9 \\
& =\mathfrak{\Im}+12 m-9
\end{aligned}
$$


where $\mathfrak{J}=\left|\lambda_{1}\right|+\left|\lambda_{2}\right|+\left|\lambda_{3}\right|$.

Theorem 19. The distance energy of $D_{6}^{m}$ is $18 m-14+\mathfrak{I}$, where $\mathfrak{I}$ is the sum of the absolute values of the roots of the equation $\quad \lambda^{3}-(18 m-14) \lambda^{2}-\left(14 m^{2}+43 m-16\right) \lambda-6 m$ $(2 m+4))=0$.

Proof. The distance matrix for $D_{6}^{m}$ of order $m n-m+1$ is

The characteristic equation of the above distance matrix is

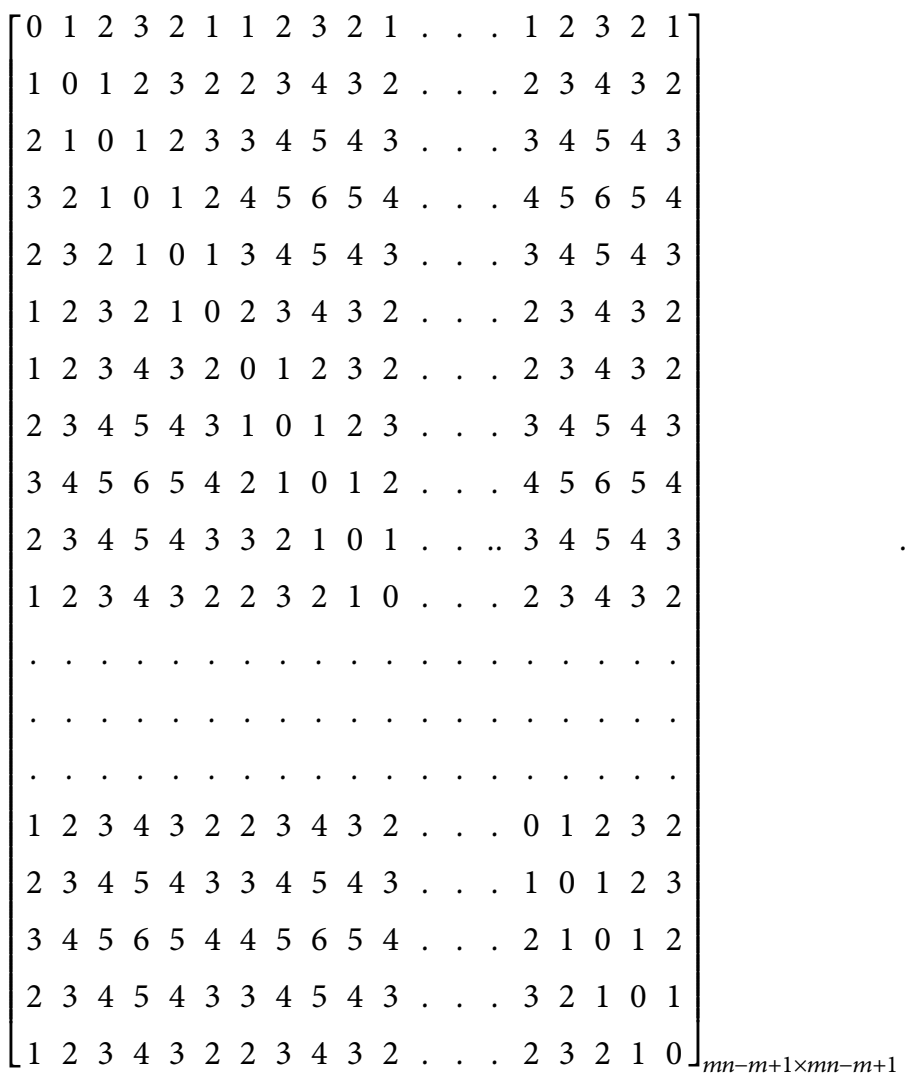

The eigenvalues of the above distance matrix are $\lambda_{1}=0$ (2m times), $\quad \lambda_{2}=-4$ ( $m$ times $), \quad \lambda_{3}=-7+\sqrt{33}$ ( $m-1$ times), $\lambda_{4}=-7-\sqrt{33}\left(m-1\right.$ times), and $\lambda_{5}, \lambda_{6}, \lambda_{7}$, where $\lambda_{5}, \lambda_{6}, \lambda_{7}$ are the roots of the cubic equation given in the following:

$$
(\lambda-0)^{2 m}(\lambda+4)^{m}\left(\lambda^{2}+14 \lambda+16\right)^{m-1}\left(\lambda^{3}-(18 m-14) \lambda^{2}-\left(14 m^{2}+43 m-16\right) \lambda-6 m(2 m+4)\right)=0 .
$$$$
\left.\lambda^{3}-(18 m-14) \lambda^{2}-\left(14 m^{2}+43 m-16\right) \lambda-6 m(2 m+4)\right)=0 .
$$

Now, distance energy can be calculated by using Definition 2.4.3 as follows:

$$
\begin{aligned}
\text { D.E. }\left(D_{6}^{m}\right) & =2 m\left|\lambda_{1}\right|+m\left|\lambda_{2}\right|+(m-1)\left|\lambda_{3}\right|+(m-1)\left|\lambda_{4}\right|+\left|\lambda_{5}\right|+\left|\lambda_{6}\right|+\left|\lambda_{7}\right| \\
& =2 m|0|+m|-4|+(m-1)|-7+\sqrt{33}|+(m-1)|-7-\sqrt{33}|+\left|\lambda_{5}\right|+\left|\lambda_{6}\right|+\left|\lambda_{7}\right| \\
& =2 m|0|+m|-4|+(m-1)|-7+\sqrt{33}|+(m-1)|-7-\sqrt{33}|+\left|\lambda_{5}\right|+\left|\lambda_{6}\right|+\left|\lambda_{7}\right| \\
& =2 m|0|+m|-4|+(m-1)|-7+\sqrt{33}|+(m-1)|-7-\sqrt{33}|+\left|\lambda_{5}\right|+\left|\lambda_{6}\right|+\left|\lambda_{7}\right|
\end{aligned}
$$




$$
\begin{aligned}
& =4 m+(m-1)(7-\sqrt{33})+(m-1)(7+\sqrt{33})+\left|\lambda_{5}\right|+\left|\lambda_{6}\right|+\left|\lambda_{7}\right| \\
& =4 m+7 m-7-\sqrt{33} m+\sqrt{33}+7 m-7+\sqrt{33} m-\sqrt{33}+\left|\lambda_{5}\right|+\left|\lambda_{6}\right|+\left|\lambda_{7}\right| \\
& =18 m-14+\mathfrak{J},
\end{aligned}
$$

where $\Im=\left|\lambda_{5}\right|+\left|\lambda_{6}\right|+\left|\lambda_{7}\right|$.

$$
H . E(G)>0.5+2 \sqrt{4 n^{2}+33 n+1} \text {. }
$$

Theorem 20. Let $G$ be a double-wheel graph; then, Harary energy is given as follows:
Proof. A Harary matrix of order $2 n+1$ for a double-wheel graph $W_{n}$ is given as follows:

$$
H\left(W_{n}\right)=\left[\begin{array}{cccccccccccccc}
0 & 1 & 1 / 2 & 1 / 2 & . & . & . & 1 & 1 / 2 & 1 / 2 & . & . & 1 / 2 & 1 \\
1 & 0 & 1 & 1 / 2 & . & . & . & 1 / 2 & 1 / 2 & 1 / 2 & . & . & 1 / 2 & 1 \\
1 / 2 & 1 & 0 & 1 & . & . & . & 1 / 2 & 1 / 2 & 1 / 2 & . & . & 1 / 2 & 1 \\
1 / 2 & 1 / 2 & 1 & 0 & . & . & . & 1 / 2 & 1 / 2 & 1 / 2 & . & . & 1 / 2 & 1 \\
. & . & . & . & . & . & . & . & . & . & . & . & . & . \\
. & . & . & . & . & . & . & . & . & . & . & . & . & . \\
. & . & . & . & . & . & . & . & . & . & . & . & . & . \\
1 & 1 / 2 & 1 / 2 & 1 / 2 & . & . & . & 0 & 1 / 2 & 1 / 2 & . & . & 1 / 2 & 1 \\
1 / 2 & 1 / 2 & 1 / 2 & 1 / 2 & . & . & . & 1 / 2 & 0 & 1 & . & . & 1 & 1 \\
1 / 2 & 1 / 2 & 1 / 2 & 1 / 2 & . & . & . & 1 / 2 & 1 & 0 & . & . & 1 / 2 & 1 \\
. & . & . & . & . & . & . & . & . & . & . & . & . & . \\
. & . & . & . & . & . & . & . & . & . & . & . & . & . \\
1 / 2 & 1 / 2 & 1 / 2 & 1 / 2 & . & . & . & 1 / 2 & 1 & 1 / 2 & . & . & 0 & 1 \\
1 & 1 & 1 & 1 & . & . & . & 1 & 1 & 1 & . & . & 1 & 0
\end{array}\right]_{2 n+1 \times 2 n+1}
$$

The characteristic equation of the Harary matrix is given as follows:

$$
\begin{aligned}
\operatorname{det}(A-\lambda I) & =0, \\
(\lambda-0.5)\left(\lambda^{2}-\frac{2 n+1}{2} \lambda-2 n\right)\left(\lambda^{2 n-2}+\lambda^{2 n-1}+\cdots \lambda\right) & =0 .
\end{aligned}
$$

The characteristic equation of this matrix has degree $2 n+1$. So, $\lambda_{1}, \lambda_{2}, \ldots \lambda_{2 n+1}$ are the eigenvalues of the given Harary matrix. The Harary energy is defined as

$$
H . E(G)=\left|\lambda_{1}\right|+\left|\lambda_{2}\right|+\left|\lambda_{3}\right|+\cdots+\left|\lambda_{2 n+1}\right| .
$$

Out of these $2 n+1$ eigenvalues, three eigenvalues are 0.5 , $(2 n+1)+\sqrt{4 n^{2}+33 n+1}$, and $(2 n+1)-\sqrt{4 n^{2}+33 n+1}$. $\left|\lambda_{3}\right|$

Clearly, $\quad\left|\lambda_{1}\right|+\left|\lambda_{2}\right|+\left|\lambda_{3}\right|+\cdots \cdots+\left|\lambda_{2 n+1}\right|>\left|\lambda_{1}\right|+\left|\lambda_{2}\right|+$

$$
=0.5+2 \sqrt{4 n^{2}+33 n+1}
$$

So, $H . E(G)>0.5+2 \sqrt{4 n^{2}+33 n+1}$.

\section{Conclusions}

In this paper, we find different energies, for example, color energy, distance energy, Laplacian energy, and Seidel energy, for the Dutch windmill graph of cycle lengths 4,5 , and 6 . Also, we find the lower bounds of the double-wheel graph for energy, Seidel energy, color energy, distance energy, Laplacian energy, and Harary energy. It is interesting to find out the computed energies for other well-known families of graphs. It is also interesting to compute other energies for the same families of graphs.

\section{Data Availability}

All the data required for this research are included within this paper.

\section{Conflicts of Interest}

The authors declare that they do not have conflicts of interest. 


\section{Authors' Contributions}

Jing $\mathrm{Wu}$ wrote the final version of the paper, used software to verify the results, and arranged funding for this paper. Muhammad Arfan Ali wrote the first version of the paper. Hafiz Mutee ur Rehman proposed the problem and supervised this work. Yan Dou reproved, analyzed, and verified all the results.

\section{Acknowledgments}

This work was sponsored in part by (1) National Natural Science Foundation of China (11501436); (2) Scientific Research Project of Shanxi Provincial Education Department (20JK0963); (3) Xijing College Fund Project (XJ200102).

\section{References}

[1] I. Gutman, "The energy of a graph," Ber. Math. Statist. Sekt. Forschungszenturm Graz, vol. 103, pp. 1-22, 1978.

[2] B. J. McClelland, "Properties of the latent roots of a matrix: the estimation of $\pi$-electron energies," The Journal of Chemical Physics, vol. 54, no. 2, pp. 640-643, 1971.

[3] P. Nageswari and P. B. Sarasija, "Edge energy bounds of graphs," Applied Mathematical Sciences, vol. 8, no. 43, pp. 2101-2104, 2014.

[4] K. C. Das and I. Gutman, "Bounds for the energy of graphs," Hacettepe Journal of Mathematics and Statistics, vol. 45, no. 3, pp. 695-703, 2016.

[5] C. Adiga and B. R. Rakshith, "Upper bounds for the extended energy of graphs and some extended equienergetic graphs," Opuscula Mathematica, vol. 38, no. 1, pp. 5-13, 2018.

[6] A. Jahanbani, "Lower bounds for the energy of graphs," AKCE International Journal of Graphs and Combinatorics, vol. 15, no. 1, pp. 88-96, 2018.

[7] W. H. Haemers, "Seidel switching and graph energy," MATCH Communications in Mathematical and in Computer Chemistry, vol. 68, no. 3, 2012.

[8] B. Zhou, "Energy of a graph," MATCH Communications in Mathematical and in Computer Chemistry, vol. 51, pp. 111118, 2004.

[9] G. Indulal, I. Gutmanb, and A. Vijayakumar, "On distance energy of graphs," MATCH Communications in Mathematical and in Computer Chemistry, vol. 60, no. 2, 2010.

[10] C. Adiga and M. Smitha, "On maximum degree energy of a graph," International Journal of Contemporary Mathematical Sciences, vol. 4, no. 8, pp. 385-396, 2009.

[11] M. R. Farahani, M. R. Kanna, and R. Pradeep, "Specific energies of friendship graph," Asian Academic Research Journal of Multidisciplinary, vol. 3, no. 1, 2016.

[12] A. D. Güngör and A. S. Cevik, "On the Harary energy and Harary Estrada index of a graph," MATCH Communications in Mathematical and in Computer Chemistry, vol. 64, no. 1, pp. 281-296, 2010.

[13] I. Gutman, X. Li, and J. Zhang, "Graph energy," in Analysis of Complex Networks, pp. 145-174, Wiley, Hoboken, NJ, USA, 2009.

[14] A. Farooq, M. Habib, A. Mahboob, W. Nazeer, and S. M. Kang, "Zagreb polynomials and redefined zagreb indices of dendrimers and polyomino chains," Open Chemistry, vol. 17, no. 1, pp. 1374-1381, 2019.

[15] S. M. Kang, M. Yousaf, M. A. Zahid, M. Younas, and W. Nazeer, "Zagreb Polynomials and redefined Zagreb indices of nanostar dendrimers," Open Physics, vol. 17, no. 1, pp. 31-40, 2019.

[16] Y. C. Kwun, A. Farooq, W. Nazeer, Z. Zahid, S. Noreen, and S. M. Kang, "Computations of the M-polynomials and degreebased topological indices for dendrimers and polyomino chains," International Journal of Analytical Chemistry, vol. 2018, Article ID 1709073, 2018.

[17] D. Wang, H. Ahmad, and W. Nazeer, "Hosoya and Harary polynomials of TUC 4 nanotube," Mathematical Methods in the Applied Sciences, 2020. 